\title{
SHELLS OF TWISTED FLAG VARIETIES AND THE ROST INVARIANT
}

\author{
S. GARIBALDI, V. PETROV, AND N. SEMENOV
}

\begin{abstract}
We introduce two new general methods to compute the Chow motives of twisted flag varieties and settle a 20-year-old conjecture of Markus Rost about the Rost invariant for groups of type $\mathrm{E}_{7}$.
\end{abstract}

\section{Contents}

1. Introduction

2. Background on algebraic groups and motives

3. Karpenko's theorem on upper motives and generic points of motives

4. Shells

5. Multiplication and Steenrod operations 10

6. Chernousov-Merkurjev formula 14

7. Weak special correspondences 16

8. Applications to motives of twisted flag varieties: type $\mathrm{E}_{6} \quad 18$

9. Reduction to characteristic zero 24

10. Applications to the Rost invariant 26

References $\quad 36$

\section{INTRODUCTION}

A twisted flag variety over an arbitrary field — such as a quadric or a SeveriBrauer variety - "typically" has no rational points. In such a case, the Chow motive of the variety provides one of the few tools for studying its geometry. In this article, we introduce two new general methods to compute the Chow motive of such a variety.

Chow motives were introduced by Alexander Grothendieck, and they have since become a fundamental tool for investigating the structure of algebraic varieties. Computing Chow motives has also proved to be valuable for addressing questions on other topics. As examples, we mention:

- Voevodsky's proof of the Milnor conjecture relied on Rost's computation of the motive of a Pfister quadric.

- Progress on the long-standing Kaplansky problem about possible values of the $u$-invariant relied on the Chow motives and Chow rings of quadrics and quadratic Grassmannians, see [Vi07].

2010 Mathematics Subject Classification. 20G15, 14C15, $20 \mathrm{G} 41$.

Key words and phrases. linear algebraic groups, twisted flag varieties, Rost invariant, Chow motives, equivariant Chow groups. 
- The structure of the powers of the fundamental ideal in Witt rings ([Ka04]), excellent connections in the motives of quadrics ([Vi10]), the proof of the hyperbolicity conjecture for orthogonal involutions ([Ka10]), the proof of Hoffmann's conjecture on the higher Witt indexes of quadratic forms ([Ka03]), an incompressibility results as in [Ka13] rely on analyzing the Chow motive of generalized Severi-Brauer varieties and products of quadrics.

- The motive of the Borel variety for $\mathrm{E}_{8}$ as in [PSZ] and [PS12] was used to answer a question of Serre [Sem] and other questions about finite subgroups of $\mathrm{E}_{8}$ [GaSe].

- Karpenko and others proved many results on isotropy of involutions on central simple algebras by analyzing motivic decompositions of projective homogeneous varieties, culminating in the paper [KaZ] by Karpenko and Zhykhovich which also treats unitary Grassmannians.

We illustrate the power of the general methods developed in this paper by calculating the Chow motives of some twisted flag varieties for which this computation was previously not possible.

First method: shells. Our first method $(\S 4)$ generalizes Vishik's shells of quadratic forms from [Vi03] and extends Karpenko's result on the upper motives from [Ka13]. Karpenko proved that any indecomposable direct summand of the Chow motive of a twisted flag variety of inner type is isomorphic to a Tate twist of the upper motive of another twisted flag variety. Thus, the study of motivic decompositions of twisted flag varieties is reduced to the study of the upper motives. For generically split varieties, the structure of upper motives was determined in [PSZ], but apart from this case there are unfortunately very few results about the structure of upper motives.

Our method is aimed to address this lack. It turns out that one can subdivide algebraic cycles on twisted flag varieties into several classes, called shells. Direct summands of the Chow motives of twisted flag varieties starting in the same shell are of the same nature, and our first main result (Theorem 4.10) asserts that one can shift these direct summands inside shells under the condition of existence of a certain cycle $\alpha$. This condition is essential; the theorem does not hold without it. Moreover, this condition is automatically satisfied for quadrics, so the notion of shell given here generalizes Vishik's shells for quadrics.

We use the shell method to prove that certain "big" direct summands are indecomposable.

Second method. Our second method (Theorem 6.4) is based on a formula of Chernousov and Merkurjev from [CM]. This method provides a broad generalization of the generic point diagram, which is a standard tool in the theory of Chow motives. Namely, if $\alpha$ is a cycle on a twisted flag $G$-variety $\bar{X}$, which is defined over the function field of a twisted flag $G$-variety $X^{\prime}$ (where $G$ is a semisimple algebraic group), then the cycle $\alpha \times 1+\beta$ on the product $\bar{X} \times \bar{X}^{\prime}$ is defined over the base field for a certain cycle $\beta$. Unfortunately, the generic point diagram does not give a precise formula for $\beta$, and one works with undefined coefficients. Our method allows one to compute $\beta$ explicitly - see Example 6.5 for an illustration. Moreover, our result provides an explicit algorithmic description of all rational cycles on the product $\bar{X} \times \bar{X}^{\prime}$, and in particular of all rational projectors (by taking $X=X^{\prime}$ ). 
We use this method in Section 8 to construct new projectors for Chow motives of $\mathrm{E}_{6}$-varieties. We do not know any alternative way of doing this.

Our two methods are complementary to each other. The first method is designed to eliminate certain motivic decomposition types, and the second one to prove that the remaining decomposition types are realizable.

Algorithm. We also provide in section 5 an algorithm to calculate the multiplication table for the equivariant and ordinary Chow rings of twisted flag varieties and the equivariant and ordinary Steenrod operations modulo $p$. This algorithm is a generalization of one described by Knutson and Tao in [KnT] for Grassmannians. This section of the paper can be read independently of the rest.

Applications. To demonstrate our methods, we provide a complete classification of motivic decompositions of all twisted flag varieties of inner type $\mathrm{E}_{6}$ (see Section 8).

We also use our methods to prove results relating isotropy of certain groups with values of the Rost invariant. Recall that the Rost invariant is a map

$$
r_{G}: H^{1}(k, G) \rightarrow H^{3}(k, \mathbb{Q} / \mathbb{Z}(2))
$$

that is functorial in $k$ and defined for every simple simply connected algebraic group $G$. For such $G$, it is, roughly speaking, the first nonzero invariant [KMRT, §31], and it generates the group of invariants with codomain $H^{3}(*, \mathbb{Q} / \mathbb{Z}(2))$ [GaMS]. It plays an important role in the study of quadratic forms (where it is known as the Arason invariant) and it was crucial in Bayer and Parimala's proof of the Hasse Principle Conjecture II for classical groups in [BP] and the proof of all known cases of the conjecture for exceptional groups. Generally speaking, such cohomological invariants are important because they provide a way to transform questions about the pointed set $H^{1}(k, G)$ into questions about an abelian group like $H^{3}(k, \mathbb{Q} / \mathbb{Z}(2))$. For the definition and basic properties of the Rost invariant, see the portion of the book [GaMS] written by Merkurjev and Garibaldi.

More than 20 years ago, Markus Rost conjectured $^{1}$ that the Rost invariant for groups of type $\mathrm{E}_{7}$ detects rationality of parabolic subgroups. We combine the motivic techniques developed here to prove his conjecture, which appears as Theorem 10.10 below. Moreover, Tonny Springer raised the general question of possible relations between the Rost invariant and rationality of parabolic subgroups for groups of type $\mathrm{E}_{7}$ in [Sp06]; Theorems 10.10 and 10.18 settle this.

We prove analogous results for all parabolic subgroups of groups of type $\mathrm{E}_{l}$, including $\mathrm{E}_{8}$ at the prime 3 (Theorems 10.10, 10.18, and 10.23). Note that usually results for primes bigger than 2 are substantially more complicated than the version for 2. Our methods work in the same manner for all primes.

Further, our results on the Rost invariant give some classification results for algebraic groups over function fields of $p$-adic curves (Corollaries 10.17 and 10.25).

We remark that we do not use the second method to prove these results about the Rost invariant, nor do we need the full generality of shells. (The full generality of both methods is essential, however, for the decomposition of the $\mathrm{E}_{6}$-varieties in Section 8.) For our Rost invariant results, it suffices to use just the first shell, the algorithm to compute the Steenrod operations (applied only to $\mathrm{E}_{7}$-varieties), Karpenko's upper motive, and Proposition 3.2 below due to De Clercq (which is a

\footnotetext{
${ }^{1}$ Letter to Jean-Pierre Serre, dated November 1992.
} 
generalization of Karpenko's [Ka10, Prop. 4.6]). On the other hand, we believe that we would not have found any of the proofs in the present article without developing the general methods first.

In summary, the applications to groups of type $\mathrm{E}_{l}$ are substantially stronger than previous results, which were obtained using algebraic and cohomological techniques. Furthermore, our proofs are more "conceptual" in that the difficult work is part of the new motivic techniques, which are completely general, as opposed to being specific to the group. Also, all results of the present article apart from a few technical statements hold over fields of any characteristic, even if the characteristic equals a torsion prime of the group.

Finally, our methods are also new for groups of classical types, and can be applied to study, for example, the generalized Severi-Brauer varieties and quadratic or symplectic Grassmannians.

\section{BACKGROUND ON ALGEBRAIC GROUPS AND MOTIVES}

Algebraic groups. Detailed information on algebraic groups can be found in [Sp98] and [KMRT].

2.1. Let $k$ be a field and $G$ a semisimple linear algebraic group of inner type over $k$. We write $\Phi$ for the root system of $G, \Phi^{+}$resp. $\Phi^{-}$for the set of positive resp. negative roots, and $\Delta$ for the Dynkin diagram of $G$ and by abuse of notation also for the set of vertices or simple roots. We enumerate the simple roots following Bourbaki, and we recall the precise numbering for groups of type $\mathrm{E}$ in (8.1) and (10.1) below.

For every subset $\Theta$ of $\Delta$, there is a projective homogeneous $G$-variety $X_{\Theta}$ of parabolic subgroups of type $\Theta$; these are the twisted flag varieties of $G$. We normalize the notation so that $X_{\emptyset}=\operatorname{Spec} k, X_{\{\alpha\}}$ corresponds to a maximal parabolic subgroup, and $X_{\Delta}$ is the Borel variety. We occasionally omit the braces and write $X_{1,2}$ for $X_{\{1,2\}}$, for example. If $G$ is a split group, then in the same way we write $P_{\Theta}$ for a standard parabolic subgroup of type $\Theta$ so that $X_{\Theta} \simeq G / P_{\Theta}$.

2.2. The Tits index of the group $G$ is the set of vertices $i \in \Delta$ such that the variety $X_{i}$ has a rational point; we draw it by circling those vertices in the Dynkin diagram of $G$. The possible Tits indexes have been determined in [Ti66], or see [Sp98, $§ 17]$ or [PSt].

Let $S$ be a maximal $k$-split torus of $G$ and following [Ti66] put $G_{\text {an }}$ for the derived subgroup $\left[Z_{G}(S), Z_{G}(S)\right]$ of the reductive group $Z_{G}(S)$. The subgroup $G_{\text {an }}$ is called the semisimple anisotropic kernel of $G$. It is semisimple and $k$-anisotropic, and it is uniquely determined by $G$ up to $G(k)$-conjugacy because the maximal $k$-split tori are conjugate under $G(k)$. The Dynkin type of $G_{\text {an }}$ equals $\Delta \backslash L$, where $L$ is the Tits index of $G$, and the Tits index of $G_{\text {an }}$ is empty.

Rost invariant. The Rost invariant from (1.1) takes values in the group $H^{3}(k, \mathbb{Q} / \mathbb{Z}(2))$, which is defined to be the direct sum over all primes $p$ of $\lim _{m} H^{3}\left(k, \mathbb{Z} / p^{m} \mathbb{Z}(2)\right)$, where

$$
H^{d+1}\left(k, \mathbb{Z} / p^{m} \mathbb{Z}(d)\right):= \begin{cases}H^{d+1}\left(k, \mu_{p^{m}}^{\otimes d}\right) & \text { if char } k \neq p ; \\ H^{1}\left(k, K_{d}\left(k_{\mathrm{sep}}\right) / p^{m}\right) & \text { if char } k=p,\end{cases}
$$

the groups on the right are Galois cohomology, and $k_{\text {sep }}$ is a separable closure of $k$, see [GaMS, pp. 151-154]. One defines $H^{3}(k, \mathbb{Z} / n \mathbb{Z}(2))$ analogously for composite $n$, and it is naturally identified with the $n$-torsion in $H^{3}(k, \mathbb{Q} / \mathbb{Z}(2))$. Note 
that $H^{1}(k, \mathbb{Z} / n \mathbb{Z}(0))$ is the Galois cohomology group $H^{1}(k, \mathbb{Z} / n \mathbb{Z})$ regardless of the characteristic of $k$.

There is a cup product map

$$
\left(\times{ }^{d} K_{1}(k)\right) \times H^{1}(k, \mathbb{Z} / n \mathbb{Z}) \rightarrow H^{d+1}(k, \mathbb{Z} / n \mathbb{Z}(d)),
$$

and we call elements of the image (including zero) symbols.

\section{Chow motives.}

2.3. By a variety we always mean a reduced separated scheme of finite type over a field. Let $p$ be a prime number. For a smooth projective variety $X$ over $k$, we write $\mathrm{CH}(X)$ for its Chow ring modulo rational equivalence and set $\mathrm{Ch}(X):=$ $\mathrm{CH}(X) \otimes \mathbb{F}_{p}$. We write deg for the degree map $\mathrm{CH}_{0}(X) \rightarrow \mathbb{Z}$, and for a field extension $K / k$ we write $X_{K}$ for the corresponding extension of scalars. A cycle $\alpha \in \operatorname{Ch}\left(X_{K}\right)$ is called rational (with respect to $k$ ), if it lies in the image of the restriction map $\mathrm{Ch}(X) \rightarrow \mathrm{Ch}\left(X_{K}\right)$. A subgroup of $\mathrm{Ch}\left(X_{K}\right)$ is called rational if all its elements are rational.

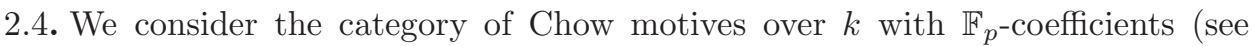
[Ma] or [EKM, §64]). The motive of a variety $X$ is denoted by $\mathscr{M}(X)$. For a field extension $K / k$ and a motive $M$ we denote by $M_{K}$ the respective extension of scalars. The shifts of Tate motives are denoted by $\mathbb{F}_{p}(i)$.

2.5. Let $X$ be a smooth projective irreducible variety over $k$. A motive $M=(X, \pi)$ for a projector $\pi$ is called geometrically (resp., generically) split, if over some field extension $F$ of $k$ (resp., over $k(X))$ it is isomorphic to a finite sum $\bigoplus_{i \in I} \mathbb{F}_{p}(i)$ of Tate motives for some multiset of non-negative indexes $I$. The field $F$ is called a splitting field of $M$, and for a cycle $\alpha \in \operatorname{Ch}(X)$ we set $\bar{\alpha}=\alpha_{F}$.

For a twisted flag variety $X$, the motive $\mathscr{M}(X)$ is geometrically split (see [Kö, Theorem 2.1]), and we denote by $\bar{X}$ the scalar extension of $X$ to a splitting field of its motive. The Chow ring of $\bar{X}$ is independent of the choice of splitting field. Its structure is explicitly described in Section 5 .

We define the Poincaré polynomial of a geometrically split motive $M$ by the formula $P(M, t)=\sum_{i \in I} t^{i} \in \mathbb{Z}[t]$. The Poincaré polynomial is independent of the choice of a splitting field $F$. We define the dimension of $M$ to be $\operatorname{dim} M:=$ $\max I-\min I$. An explicit formula for $P(\mathscr{M}(X), t)$ for a twisted flag variety $X$ is given in $[\mathrm{PS} 10, \S 2]$.

In a similar way we define the Poincaré polynomial of a finite-dimensional $\mathbb{Z}_{\geq 0^{-}}$ graded vector space $A^{*}$ as $P\left(A^{*}, t\right)=\sum_{i \geq 0} \operatorname{dim} A^{i} \cdot t^{i}$.

2.6 (Krull-Schmidt). For a motive $M$ we say that the Krull-Schmidt theorem holds for $M$, if for any two motivic decompositions of $M$

$$
M \simeq M_{1} \oplus \ldots \oplus M_{a} \simeq N_{1} \oplus \ldots \oplus N_{b}
$$

with all motives $M_{i}, N_{j}$ indecomposable, we have $a=b$ and there exists a permutation $\sigma$ of $\{1, \ldots, a\}$ such that $M_{i} \simeq N_{\sigma(i)}$ for all $i=1, \ldots, a$.

By [CM] the Krull-Schmidt theorem holds for all twisted flag varieties in the category of motives with $\mathbb{F}_{p}$-coefficients. 


\section{KARPENKO'S THEOREM ON UPPER MOTIVES AND GENERIC POINTS OF MOTIVES}

Let $G$ be a semisimple linear algebraic group of inner type over a field $k, X$ be a twisted $G$-homogeneous flag variety over $k, p$ a prime number, and $\mathcal{U}(X)$ the (unique) indecomposable direct summand of the Chow motive of $X$ with $\mathbb{F}_{p^{-}}$ coefficients such that $\mathrm{Ch}^{0}(\mathcal{U}(X)) \neq 0$. The set of isomorphism classes of the motives $\mathcal{U}(Y)$ for all twisted $G$-homogeneous flag varieties $Y$ is called the set of upper motives of $G$.

3.1. Proposition (Karpenko, [Ka13, Theorem 3.5]). Every indecomposable summand of $X$ is isomorphic to a Tate shift of an upper motive $\mathcal{U}(Y)$, where $Y$ runs over all twisted $G$-homogeneous flag varieties over $k$ such that the Tits index of $G_{k(Y)}$ contains the Tits index of $G_{k(X)}$.

We also need a result of De Clercq:

3.2. Proposition (De Clercq, [DC, Thm. 1.1]). Let $X$ and $Y$ be twisted flag varieties, and let $M$ and $N$ be direct summands of $\mathscr{M}(X)$ and $\mathscr{M}(Y)$ respectively. If $N_{k(X)}$ is an indecomposable direct summand of $M_{k(X)}$ and every cycle in $\mathrm{Ch}(\overline{Y \times X})$ which is defined over $k(X)\left(Y_{k(X)}\right)$ is defined over $k(Y)$, then $N$ is a direct summand of $M$.

3.3. Definition. Let now $X$ be a smooth projective irreducible variety and $M=$ $(X, \pi)$ a geometrically split motive. Assume that over a splitting field $F$ of $M$ the motive $M_{F} \simeq \bigoplus_{i \in I \cup\{l\}} \mathbb{F}_{p}(i)$ for a multiset $I$ of indexes such that every $i \in I$ is bigger than $l$. Then $\mathrm{Ch}^{l}\left(M_{F}\right) \simeq \mathbb{F}_{p}$, and any nonzero element in $\mathrm{Ch}^{l}\left(M_{F}\right)$ is called a generic point of $M$; we abuse language and write "the" generic point.

The following two results are well-known:

3.4. Lemma. Let $X$ be a twisted flag G-variety. The generic point of a direct summand of the motive of $X$ is rational (i.e., defined over $k$ ).

Proof. Follows from Prop. 3.1.

3.5. Lemma. If, in the notation of Definition 3.3, $M$ is generically split, then the generic point of $M$ is rational.

Proof. Let in the notation of Definition 3.3 $M=(X, \pi)$. The motive $M_{k(X)}$ is a direct sum of shifted Tate motives. Let $l$ be the smallest integer such that the Tate motive $\mathbb{F}_{p}(l)$ is a direct summand of $M_{k(X)}$. This Tate motive is defined by two cycles $a \in \mathrm{Ch}_{l}\left(X_{k(X)}\right)$ and $b \in \mathrm{Ch}^{l}\left(M_{k(X)}\right)$ with $\operatorname{deg}(a b)=1$ and in the Sweedler notation

$$
\bar{\pi}=a \times b+x_{(1)} \times x_{(2)}
$$

with $\operatorname{codim} x_{(2)}>l$. We want to show that $b$ is defined over $k$.

Consider $M_{k(X)}$. By the generic point diagram ([PSZ, Lemma 1.8]) the cycle

$$
b \times 1+y_{(1)} \times y_{(2)}
$$

with $\operatorname{codim} y_{(1)}<l$ is rational. Hence, the product of the above cycles equals

$$
\text { pt } \times b+z_{(1)} \times z_{(2)},
$$


where $\operatorname{codim} z_{(1)}<\operatorname{dim} X$ and pt is the class of a rational point on $X$, and is rational. Taking the push-forward with respect to the second projection

$$
X \times X \rightarrow X,
$$

one sees that $b$ is rational.

\section{SHELlS}

The content of this section is a generalization of the notion of shells for quadratic forms invented by Vishik (see [Vi03]) and Karpenko's result quoted as Proposition 3.1 above. Let $X$ denote a twisted $G$-homogeneous flag variety that is not a point.

4.1. Definition (big shells). For each set $\Psi$ of vertices of the Dynkin diagram $\Delta$ of $G$, we put $K_{\Psi}$ for the function field of the variety $X_{\Psi}$. Define the (big) shell $\mathrm{SH}_{\leqslant \Psi}$ of $X$ to be the union for all $i$ of the $b \in \mathrm{Ch}^{i}(\bar{X})$ such that

(1) $b$ is defined over $K_{\Psi}$ and

(2) there is an $a \in \mathrm{Ch}_{i}(\bar{X})$ defined over $K_{\Psi}$ such that $\operatorname{deg}(a b)=1 \in \mathbb{F}_{p}$.

Note that each shell is closed under multiplication by elements of $\mathbb{F}_{p}^{\times}$, and that the shells depend on the prime $p$ (even though the Poincaré polynomial of $\mathrm{Ch}(\bar{X})$ does not).

4.2. Examples. A shell $\mathrm{SH}_{\leqslant \Psi}$ is nonempty iff $X \times K_{\Psi}$ has a zero-cycle of degree not divisible by $p$. Consequently, for $\Theta \subseteq \Delta$ such that $X=X_{\Theta}$, the shell $\mathrm{SH}_{\leq \Theta}$ is always nonempty. We call it the first shell of $X$.

For $\Psi=\Delta$ or more generally for any $\Psi$ such that $K_{\Psi}$ splits $G, \mathrm{SH}_{\leqslant \Psi}$ is the set of nonzero homogeneous elements of $\operatorname{Ch}(\bar{X})$, because the pairing $(a, b) \mapsto \operatorname{deg}(a b)$ on $\operatorname{Ch}(\bar{X})$ is non-degenerate. We call this the last shell of $X$.

4.3. Example. If $\Psi \subseteq \Psi^{\prime}$, then there is a natural surjection $X_{\Psi^{\prime}} \rightarrow X_{\Psi}$, hence an inclusion $K_{\Psi} \subseteq K_{\Psi^{\prime}}$, so $\mathrm{SH}_{\leqslant \Psi} \subseteq \mathrm{SH}_{\leqslant \Psi^{\prime}}$.

4.4. Definition. A $(p, \Theta)$-index $S$ of $G$ is a set $\Theta \subseteq S \subseteq \Delta$ such that there exists an extension $L$ of $k$ with the properties that (a) the Tits index of $G_{L}$ is $S$ and (b) every finite extension of $L$ has degree a power of $p$.

The set of $(p, \Theta)$-indexes of $G$ is a partially ordered set (by inclusion). We define the height of $X=X_{\Theta}$ as the maximal number of elements in a chain of $(p, \Theta)$-indexes of $G$.

4.5. Proposition. Let $\left\{S_{i} \mid i \in I\right\}$ be the set of $(p, \Theta)$-indexes of $G$.

(1) Every nonempty shell on $X=X_{\Theta}$ equals $\mathrm{SH}_{\leqslant n_{j} \in J} S_{j}$ for some subset $J \subseteq I$.

(2) Suppose that $\Theta \subseteq \Psi, \Psi^{\prime} \subseteq \Delta$ are such that, for every i, if $\Psi^{\prime} \subset S_{i}$ then $\Psi \subset S_{i}$. Then $\mathrm{SH}_{\leqslant \Psi} \subseteq \mathrm{SH}_{\leqslant \Psi^{\prime}}$.

Proof. First suppose that $\Psi, \Psi^{\prime} \subseteq \Delta$ are such that there exists a finite extension $F$ of $K_{\Psi^{\prime}}$ of degree not divisible by $p$ such that $X_{\Psi}(F) \neq \emptyset$. Put $E$ for the fraction field of the integral domain $F \otimes K_{\Psi}$; it is the function field of the $F$-variety $X_{\Psi} \times F$. The diagram

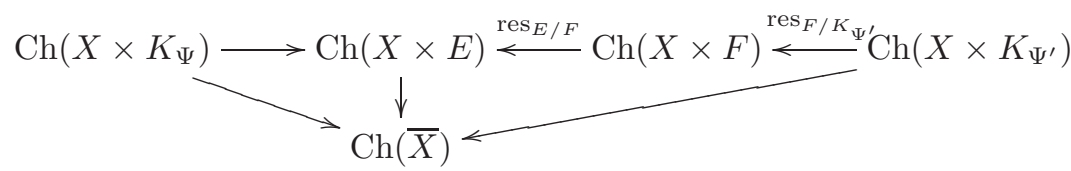


commutes where all the arrows are scalar extension. Each $b \in \mathrm{SH}_{\leqslant \Psi}$ is the image of some $b_{0} \in \operatorname{Ch}\left(X \times K_{\Psi}\right)$ and we consider the image of $b_{0}$ in $\operatorname{Ch}(X \times E)$. As $X_{\Psi}(F)$ is nonempty, $E$ is a purely transcendental extension of $F$, hence $\operatorname{res}_{E / F}$ is an isomorphism by [FlOV, Prop. 2.1.8]. The arrow $\operatorname{res}_{F / K_{\Psi}}$ is also an isomorphism, so the image of $b_{0}$ in $\mathrm{Ch}\left(X \times K_{\Psi^{\prime}}\right)$ maps to $b \in \mathrm{SH}_{\leqslant \Psi^{\prime}}$, proving that $\mathrm{SH}_{\leqslant \Psi} \subseteq \mathrm{SH}_{\leqslant \Psi^{\prime}}$.

For (2), there is a finite extension $F$ of $K_{\Psi^{\prime}}$ of degree not divisible by $p$ such that the Tits index of $G_{F}$ is $S_{i}$ for some $i$; necessarily this $S_{i}$ contains $\Psi^{\prime}$, hence also $\Psi$, i.e., $X_{\Psi}(F)$ is nonempty. The previous paragraph gives (2).

Now let $\Psi$ be an arbitrary subset of $\Delta$ such that $\mathrm{SH}_{\leqslant \Psi}$ is nonempty. Then there is a finite extension $F$ of $K_{\Psi}$ of degree not divisible by $p$ such that $X_{\Theta}(F)$ is nonempty and the Tits index of $G_{F}$ contains both $\Psi$ and $\Theta$, i.e., $X_{\Theta \cup \Psi} \times K_{\Psi}$ has a zero-cycle of degree not divisible by $p$. By the previous paragraph, we find that $\mathrm{SH}_{\leqslant \Psi}=\mathrm{SH}_{\leqslant \Psi \cup \Theta}$.

For (1), let $\Psi_{0} \subseteq \Delta$ be such that $\mathrm{SH}_{\leqslant \Psi_{0}}$ is nonempty. By the previous paragraph, we may assume that $\Psi_{0} \supseteq \Theta$. Put $\Psi$ for the intersection of all the $S_{i}$ 's containing $\Psi_{0}$. By (2), $\mathrm{SH}_{\leqslant \Psi}=\mathrm{SH}_{\leqslant \Psi_{0}}$, hence (1).

4.6. Example (Quadrics). In [Vi03] Vishik describes a subdivision of the Chow group of projective quadrics into shells.

Let $p=2$ and let $q$ be an anisotropic regular quadratic form over $k$ of dimension $n+2$ and $X$ the projective quadric given by the equation $q=0$. Since in the present article we consider the groups of inner type only, we assume that the discriminant of $q$ is trivial, if $n$ is even.

Let $h \in \mathrm{Ch}^{1}(\bar{X})$ be the class of a hyperplane section of $\bar{X}$ and $l_{s}, s=0, \ldots,[n / 2]$, the classes of $s$-dimensional subspaces on $\bar{X}$. Then the Chow group $\mathrm{Ch}^{*}(\bar{X})$ has a basis

$$
\left\{h^{s}, l_{s} \mid s=0, \ldots,[n / 2]\right\} .
$$

Let $i_{1}<\cdots<i_{r}$ be the splitting pattern of $q$ (in the usual sense of [EKM, p. 104], as opposed to the variation used in [Vi03, p. 31]) and set $i_{0}=0$. Then the cycles $\left\{h^{s}, l_{s} \mid i_{t-1} \leq s \leq i_{t}-1\right\}$ belong to the shell $t \in \mathbb{N}$ in the notation of Vishik.

In our notation the cycles $\left\{h^{s}, l_{s} \mid 0 \leq s<i_{t}\right\}$ belong to $\mathrm{SH}_{\leqslant\left\{i_{t}\right\}}$, and the cycles $\left\{h^{s}, l_{s} \mid i_{t-1} \leq s<i_{t}\right\}$ belong to $\mathrm{SH}_{\leqslant\left\{i_{t}\right\}} \backslash \mathrm{SH}_{\leqslant\left\{i_{t-1}\right\}}$.

4.7. Example. Suppose the set of $(p, \Theta)$-indexes of $G$ is contained in $\left\{S_{1}, \ldots, S_{r}\right\}$ such that $S_{1} \subset S_{2} \subset \cdots \subset S_{r}=\Delta$. Then by Proposition 4.5(1), the nonempty shells of $S$ are

$$
\mathrm{SH}_{\leqslant \Theta}=\mathrm{SH}_{\leqslant S_{1}} \subseteq \mathrm{SH}_{\leqslant S_{2}} \subseteq \cdots \subseteq \mathrm{SH}_{\leqslant S_{r}}=\mathrm{SH}_{\leqslant \Delta} .
$$

This situation occurs in Example 4.6, where we find $r$ distinct $(2,\{1\})$-indexes and $r$ distinct shells.

4.8. Definition (small shells). For each set $\Psi$ of vertices of the Dynkin diagram of $G$, we set the (small) shell $\mathrm{SH}_{\Psi}$ to be the union for all $i$ of the cycles $b \in \mathrm{Ch}^{i}(\bar{X})$ such that $b$ is the generic point of an indecomposable direct summand $M$ of the motive of $X$ such that $M$ is isomorphic to a Tate twist of $\mathcal{U}\left(X_{\Psi}\right)$. In particular, by the proof of [Ka13, Th. 3.5], $\mathrm{SH}_{\Psi} \subseteq \mathrm{SH}_{\leqslant \Psi}$.

We remark that the big shells reflect the splitting behavior of the group $G$ and the small shells reflect the motivic behavior of $G$.

We say that a motive $M$ starts in the shell $\mathrm{SH}_{\Psi}$ (resp. in codimension $l$ ), if its generic point belongs to $\mathrm{SH}_{\Psi}$ (resp. to $\mathrm{Ch}^{l}(\bar{X})$ ). 
4.9. Lemma. Let $X$ be a smooth projective variety over $k$ and $M$ an indecomposable geometrically split motive with a splitting field $F$ satisfying the following conditions:

(1) the kernel of the natural map $\operatorname{End}(M) \rightarrow \operatorname{End}\left(M_{F}\right)$ consists of nilpotent correspondences;

(2) $M_{F} \simeq \bigoplus_{i \in I \cup\{l\}} \mathbb{F}_{p}(i)$ for a multiset $I$ of indexes such that every $i \in I$ is bigger than $l$;

(3) there exist two morphisms $\alpha: M \rightarrow \mathscr{M}(X)$ and $\beta: \mathscr{M}(X) \rightarrow M$ such that the composition $\beta \circ \alpha: M \rightarrow M$ maps over $F$ the generic point of $M_{F}$ identically on itself.

Then $M$ is isomorphic to a direct summand of $\mathscr{M}(X)$.

Proof. Let $M=(Y, \pi)$ for some smooth projective variety $Y$ over $k$. Since $M$ is geometrically split, the $\operatorname{ring} \operatorname{End}\left(M_{F}\right)$ is finite, and therefore some power, say $n$, of $\bar{\beta} \circ \bar{\alpha} \in \operatorname{End}\left(M_{F}\right)$ is a projector. This projector is non-zero by condition 3). Since $M$ is indecomposable, this projector must be equal to $\bar{\pi}$.

Denote $\alpha^{\prime}:=\alpha \circ(\beta \circ \alpha)^{\circ(n-1)}: M \rightarrow \mathscr{M}(X)$. Then $\bar{\beta} \circ \bar{\alpha}^{\prime}=\bar{\pi}$. By condition 1) $\beta \circ \alpha^{\prime}=\pi+\nu$ for some nilpotent correspondence $\nu$. Denote $\beta^{\prime}:=(\pi+\nu)^{-1} \circ \beta$. Then $\beta^{\prime} \circ \alpha^{\prime}=\pi$, and the lemma follows.

The following theorem is known for smooth projective quadrics by [Vi03].

4.10. Theorem. Let $b \in \mathrm{Ch}^{l}(\bar{X}) \cap \mathrm{SH}_{\Psi}$ be the generic point of an indecomposable direct summand $M$ of $\mathscr{M}(X)$ and $\alpha \in \mathrm{Ch}^{t}(\bar{X})$ a cycle defined over $k$. If the cycle $b^{\prime}:=b \cdot \alpha$ is in $\mathrm{SH}_{\leqslant \Psi}$, then there is an indecomposable direct summand $M^{\prime}$ of $\mathscr{M}(X)$ with generic point $b^{\prime}$ and isomorphic to $M(t)$.

The results of Section 8 below show that one cannot in general weaken any condition of the theorem.

Proof of Theorem 4.10. Set $Y=X_{\Psi}$. By assumptions $M$ is isomorphic to $N(l)$ for the upper motive $N=\mathcal{U}(Y)$. Let $d^{\prime}$ be a cycle dual to $b^{\prime}$ in the definition of shells. Then $d^{\prime}$ is defined over $k(Y)$.

Define a sequence of morphisms

$$
M(t)=N(t+l) \stackrel{\alpha}{\rightarrow} Y(t+l) \stackrel{\beta}{\rightarrow} X \stackrel{\gamma}{\rightarrow} X(t) \stackrel{\delta}{\rightarrow} M(t),
$$

where $\alpha$ is an embedding of $N(t+l)$ as a direct summand of $Y(t+l)$,

$$
\bar{\beta}=1 \times d^{\prime}+y_{(1)} \times x_{(2)} \in \mathrm{Ch}_{\operatorname{dim} Y+l+t}(\bar{Y} \times \bar{X})
$$

with $\operatorname{codim} y_{(1)}>0$,

$$
\bar{\gamma}=(\alpha \times 1) \cdot \Delta_{X} \in \mathrm{Ch}_{\operatorname{dim} X-t}(\bar{X} \times \bar{X}),
$$

and $\delta$ is the projection onto the direct summand.

To finish the proof it suffices to notice that the composition $\delta \circ \gamma \circ \beta \circ \alpha$ maps $\mathbb{F}_{p}(t+l)$ to $\mathbb{F}_{p}(t+l)$ identically over $\bar{k}$ and apply Lemma 4.9.

4.11. Corollary. Let $b \in \mathrm{Ch}^{l}(\bar{X})$ be a rational cycle from the first shell of $X$. Then there is an indecomposable direct summand of $X$ with generic point $b$ isomorphic to the l-th Tate shift of the upper motive of $X$. 


\section{Multiplication And Steenrod operations}

5.1. Let $G_{0}$ be a split adjoint semisimple group. We fix a parabolic subgroup $P$ containing a Borel subgroup $B$ containing a maximal split torus $T$ of $G_{0}$. Occasionally we need to perform explicit calculation in $\mathrm{CH}^{*}\left(G_{0} / P\right)$ or in $\mathrm{Ch}^{*}\left(G_{0} / P\right)$ considered as a ring or (in the latter case) as a module over the Steenrod algebra. In this section, we provide algorithms for doing so based on passing to the $T$-equivariant cohomology described in [Bri], as was done for Grassmannians in [KnT]. There is a diverse literature studying the equivariant Chow rings, of which we indicate as examples [FoK], [GKM], [HHH], [Ty], [GHZ], and the references therein.

It is well-known (see e.g. [Kö]) that $\mathrm{CH}^{*}\left(G_{0} / P\right)$ has an additive basis consisting of the classes of Schubert subvarieties $X_{w}=[\overline{B w P / P}]$, where $w \in W / W_{P}, W$ stands for the Weyl group of $G_{0}$ and $W_{P}$ stands for the Weyl group of (a Levi subgroup of) $P$. We identify the cosets in $W / W_{P}$ with their minimal representatives. The dimension of $X_{w}$ is $l(w)$, the minimal length of $w$ in the simple reflections.

Sometimes it is more convenient to enumerate the generators as

$$
Z_{w}=X_{w_{0} w w_{0}^{P}},
$$

where $w_{0}$ is the longest element of $W$ and $w_{0}^{P}$ is the longest element of $W_{P}$. Then the codimension of $Z_{w}$ is $l(w)$, in particular, we have

$$
\mathrm{pt}=X_{1}=Z_{w_{0} w_{0}^{P}}
$$

Note that $Z_{w}$ is the Poincaré dual to $X_{w}$ in the sense that

$$
X_{u} \cdot Z_{w}=\delta_{u, w} \mathrm{pt}
$$

If $Q \subseteq P$ is another parabolic subgroup, the pull-back map

$$
\mathrm{CH}^{*}\left(G_{0} / P\right) \rightarrow \mathrm{CH}^{*}\left(G_{0} / Q\right)
$$

is injective and sends $Z_{w}$ in $\mathrm{CH}^{*}\left(G_{0} / P\right)$ to $Z_{w}$ in $\mathrm{CH}^{*}\left(G_{0} / Q\right)$. Sometimes we write $Z_{\left[i_{1}, \ldots, i_{l}\right]}$ for $Z_{w}$ with $w=s_{i_{1}} \cdots s_{i_{l}}$ a reduced decomposition.

5.2. Remark (Comparison with other algorithms). There are many recipes in the literature for computing the multiplication table in the basis $Z_{w}$, a.k.a. the generalized Littlewood-Richardson coefficients, see e.g. [De]. But as far as the authors know, only the one in [DuZ] and [Du] can be adapted to computing also the Steenrod operations. It is based on the consideration of the Bott-Samelson resolution of $G_{0} / B$. This resolution is a toric variety, and the structure of its Chow ring and the structure of its Steenrod algebra are both well-known, and one finds explicit combinatorial formulas. The algorithm presented below is in terms of equivariant cohomology, so is more general than the Duan-Zhao algorithm. Also, our practical experience in performing the calculations used below in Lemma 10.8 suggests that our algorithm can be substantially faster.

5.3. Let $\widehat{T}$ be the group of characters of $T$, that is the root lattice of $G_{0}$, with basis consisting of fundamental roots $\alpha_{1}, \ldots, \alpha_{n}$. (In particular, $n$ is the rank of $G$.) The ring $\mathrm{CH}_{T}^{*}(\mathrm{pt})$ coincides with the symmetric algebra $S(\widehat{T}) \simeq \mathbb{Z}\left[\alpha_{1}, \ldots, \alpha_{n}\right]$ of $\widehat{T}$.

Observe that the pullback of the structural map gives $\mathrm{CH}_{T}^{*}\left(G_{0} / P\right)$ the structure of a $\mathrm{CH}_{T}^{*}(\mathrm{pt})$-module. We have

$$
\widehat{T} \subset \mathrm{CH}_{T}^{*}(\mathrm{pt}) \rightarrow \mathrm{CH}_{T}^{*}\left(G_{0} / P\right) \rightarrow \mathrm{CH}^{*}\left(G_{0} / P\right),
$$


the last map is surjective with kernel generated by the image of $\widehat{T}$, see [Bri, Section 2.3].

5.4. There are $T$-equivariant analogs of Schubert classes $Z_{w}^{T}$ whose images in $\mathrm{CH}^{*}\left(G_{0} / P\right)$ are $Z_{w}$. The $T$-fixed points of $G_{0} / P$ are parametrized by $W / W_{P}$, see [GHZ, Section 2]. Let $\iota_{w}$ be the pull-back map

$$
\mathrm{CH}_{T}^{*}\left(G_{0} / P\right) \rightarrow \mathrm{CH}_{T}^{*}(\mathrm{pt})
$$

induced by the inclusion of the fixed point corresponding to $w \in W / W_{P}$. Then the direct sum map

$$
\mathrm{CH}_{T}^{*}\left(G_{0} / P\right) \stackrel{\oplus_{w \in W / W_{P} \iota_{w}}}{\longrightarrow} \bigoplus_{w \in W / W_{P}} \mathrm{CH}_{T}^{*}(\mathrm{pt})
$$

is injective, see [GHZ, Theorem 2.1].

5.5. Lemma (cf. [KnT, §2]). Fix $w \in W / W_{P}$.

(1) $\iota_{u}\left(Z_{w}^{T}\right)=0$ for all $u \nsupseteq w$ in the strong Bruhat order.

(2) $\iota_{w}\left(Z_{w}^{T}\right)=\prod_{\alpha \in \Phi^{+}, w^{-1}(\alpha) \in \Phi^{-}} \alpha$.

(3) For any $x \in \mathrm{CH}_{T}^{*}\left(G_{0} / P\right)$ with $\iota_{u}(x)=0$ for all $u \nsupseteq w$, the polynomial $\iota_{w}(x)$ is divisible by $\iota_{w}\left(Z_{w}^{T}\right)$ in $\mathrm{CH}_{T}^{*}(\mathrm{pt})$.

Actually $\iota_{v}\left(Z_{w}^{T}\right)$ can be computed via the generalized Billey formula ([Ty, Theorem 7.1]). Namely, if $v=s_{i_{1}} \ldots s_{i_{l}}$ is a reduced decomposition, we have

$$
\iota_{v}\left(Z_{w}^{T}\right)=\sum_{s_{i_{j_{1}}} \ldots s_{i_{j_{k}}} \in R(w)} r\left(j_{1}\right) \ldots r\left(j_{k}\right),
$$

where $r(j)=s_{i_{1}} \ldots s_{i_{j-1}}\left(\alpha_{i_{j}}\right)$ and $R(w)$ is the set of all reduced decompositions of $w$. Properties (1) and (2) immediately follow, and (3) follows from (2) and [GHZ, (2.12)].

Now we describe an algorithm to compute the ring structure and the action of the Steenrod algebra on $\mathrm{Ch}^{*}\left(G_{0} / P\right)$.

Elimination procedure. The core of the algorithm is the following elimination procedure which takes as input $x \in \mathrm{CH}_{T}^{m}\left(G_{0} / P\right)$ and returns $a_{w} \in \mathrm{CH}_{T}^{*}(\mathrm{pt})$ for $w \in W / W_{P}$ such that $x=\sum_{w} a_{w} Z_{w}^{T}$.

5.6. Assume we are given $\iota_{w}(x)$ for all $w \in W / W_{P}$ with $l(w) \leq m$.

Extend the Bruhat order to a linear order on $W / W_{P}$. We remark that the elimination procedure (but not the final result) formally depends on the extension of the Bruhat order. Let $u \in W / W_{P}$ be the minimal element such that $\iota_{u}(x) \neq 0$. If such $u$ does not exist, then $x=0$.

Then by Lemma $5.5(3) \iota_{u}(x)$ is divisible by $\iota_{u}\left(Z_{u}^{T}\right)$. In particular, since $\operatorname{deg}\left(\iota_{u}(x)\right)=$ $m$ and $\operatorname{deg}\left(\iota_{u}\left(Z_{u}^{T}\right)\right)=l(u)$ by Lemma $5.5(2)$, we have $l(u) \leq m$.

Assume $l(u)<m$. Using the explicit formula of Lemma 5.5(2) we compute the quotient polynomial $b_{u}:=\frac{\iota_{u}(x)}{\iota_{u}\left(Z_{u}^{T}\right)}$ and set $x^{\prime}:=x-b_{u} \cdot Z_{u}^{T}$. Now we apply the same procedure to $x^{\prime}$ instead of $x$. Observe that by construction $\iota_{u}\left(x^{\prime}\right)=0$ and $\iota_{w}\left(x^{\prime}\right)=0$ for $w<u$ by Lemma 5.5(1). Therefore eventually we will arrive to the situation when either $x=0$ or $l(u)=m$. 
Consider now all $v \in W / W_{P}$ such that $l(v)=m$. Since these $v$ 's have the same length, they are incomparable in the Bruhat order. Therefore the same consideration shows that $b_{v}:=\frac{\iota_{v}(x)}{\iota_{v}\left(Z_{v}^{T}\right)}$ are integers. Set $y:=x-\sum_{l(v)=m} b_{v} Z_{v}^{T}$.

We claim that $y=0$. Indeed, assume $y \neq 0$. Let $u$ be the minimal element such that $\iota_{u}(y) \neq 0$. Then again $\iota_{u}(y)$ is divisible by $\iota_{u}\left(Z_{u}^{T}\right)$. But $\operatorname{deg}\left(\iota_{u}(y)\right)=m$ and $\operatorname{deg}\left(\iota_{u}\left(Z_{u}^{T}\right)\right)=l(u)>m$. This finishes our elimination procedure.

\section{Multiplication, Steenrod operations.}

5.7. Multiplication. Let $u, v \in W / W_{P}$. Using the elimination procedure, we compute the expansion

$$
Z_{u}^{T} \cdot Z_{v}^{T}=\sum_{w \in W / W_{P}} a_{w} Z_{w}^{T} \quad \text { with } a_{w} \in \mathrm{CH}_{T}^{*}(\mathrm{pt}) .
$$

Therefore

$$
Z_{u} \cdot Z_{v}=\sum_{w \in W / W_{P}} \bar{a}_{w} Z_{w} \text { in } \mathrm{CH}^{*}\left(G_{0} / P\right),
$$

where $\bar{a}_{w}$ is the image of $a_{w}$ under the homomorphism

$$
\mathrm{CH}_{T}^{*}(\mathrm{pt}) \rightarrow \mathrm{CH}^{*}(\mathrm{pt})=\mathbb{Z}
$$

that sends a polynomial to its constant term.

5.8. Steenrod operations. Let $p$ be a prime number and assume char $k \neq p$. For $x \in \mathrm{Ch}^{*}\left(G_{0} / P\right)$ let

$$
S^{\bullet}(x)=\sum_{j \geq 0} S^{j}(x) t^{j} \in \mathrm{Ch}^{*}\left(G_{0} / P\right)[t]
$$

denote the total Steenrod operation (see e.g. [Bro]).

Recall that $\mathrm{CH}_{T}^{*}(\mathrm{pt})$ is the polynomial ring $\mathbb{Z}\left[\alpha_{1}, \ldots, \alpha_{n}\right]$ in simple roots. Set $\mathrm{Ch}_{T}^{*}(\mathrm{pt}):=\mathrm{CH}_{T}^{*}(\mathrm{pt}) / p$.

The total Steenrod operation on $\mathrm{Ch}_{T}^{*}(\mathrm{pt})$ is given by

$$
\begin{aligned}
\mathbb{F}_{p}\left[\alpha_{1}, \ldots, \alpha_{n}\right] & \stackrel{S^{\bullet}}{\longrightarrow} \mathbb{F}_{p}\left[\alpha_{1}, \ldots, \alpha_{n}\right][t] \\
\alpha_{j} & \mapsto \alpha_{j}+t \alpha_{j}^{p} .
\end{aligned}
$$

Let $u \in W / W_{P}, j \geq 0$. Using the elimination procedure, we find the expansion

$$
S^{j}\left(Z_{u}^{T}\right)=\sum_{v \in W / W_{P}} a_{v} Z_{v}^{T} \quad \text { with } a_{v} \in \mathrm{Ch}_{T}^{*}(\mathrm{pt}) .
$$

Then

$$
S^{j}\left(Z_{u}\right)=\sum_{v \in W / W_{P}} \bar{a}_{v} Z_{v}
$$

5.9. Chern classes. There is an effective procedure to compute the $T$-equivariant Chern classes of a $G_{0}$-equivariant vector bundle $V$ on $G_{0} / P$. Indeed, the $r$-th Chern class $c_{r}^{T}(V)$ of $V$ is the image of the Chern class $c_{r}^{G_{0}}(V)$ under the map

$$
\mathrm{CH}_{G_{0}}^{*}\left(G_{0} / P\right) \rightarrow \mathrm{CH}_{T}^{*}\left(G_{0} / P\right)
$$

so it lies inside $\mathrm{CH}_{T}^{*}\left(G_{0} / P\right)^{W}$ (the Weyl group action as in [Ty, Section 4]), and its value is determined by one entry, namely,

$$
\left.\iota_{w}\left(c_{r}^{T}(V)\right)=w\left(\iota_{[}\right]\left(c_{r}^{T}(V)\right)\right)
$$


The entry at [] can be computed through the map

$$
\mathrm{CH}_{G_{0}}^{*}\left(G_{0} / P\right) \rightarrow \mathrm{CH}_{P}^{*}(\mathrm{pt}) \rightarrow \mathrm{CH}_{T}^{*}(\mathrm{pt})
$$

and it coincides with the $r$-th elementary symmetric function in roots of $V$. Then one applies the elimination procedure. We now illustrate this.

5.10. Example. Let $G_{0}$ be the split group of type $\mathrm{G}_{2}$ and $P$ its parabolic subgroup of type 2 . There are exactly two 5 -dimensional twisted $G_{0}$-homogeneous flag varieties: a projective quadric, which is the variety of parabolic subgroups of type 1 , and $G_{0} / P$ (which is not a quadric). We compute some products in $\mathrm{CH}^{*}\left(G_{0} / P\right)$.

The representatives of minimal length in $W / W_{P}$ in the (decreasing) Bruhat order are:

$$
Z_{[2,1,2,1,2]}, Z_{[1,2,1,2]}, Z_{[2,1,2]}, Z_{[1,2]}, Z_{[2]}, Z_{[]} .
$$

Put $\iota:=\oplus_{w \in W / W_{P}} \iota_{w}$.

Let us compute, say, $\iota_{[2,1,2,1,2]}\left(Z_{[2]}^{T}\right)$. By $(5.1)$ we have

$\iota_{[2,1,2,1,2]}\left(Z_{[2]}^{T}\right)=r(1)+r(3)+r(5)=\alpha_{2}+s_{1} s_{2}\left(\alpha_{1}\right)+s_{2} s_{1} s_{2} s_{1}\left(\alpha_{2}\right)=6 \alpha_{1}+4 \alpha_{2}$

Computing the other entries in the same way we get

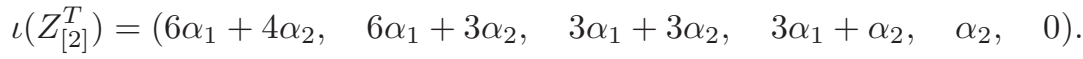

Let us compute $\left(Z_{[2]}^{T}\right)^{2}$. Squaring (5.2) we obtain:

$\iota\left(\left(Z_{[2]}^{T}\right)^{2}\right)=\left(\left(6 \alpha_{1}+4 \alpha_{2}\right)^{2}, \quad\left(6 \alpha_{1}+3 \alpha_{2}\right)^{2}, \quad\left(3 \alpha_{1}+3 \alpha_{2}\right)^{2}, \quad\left(3 \alpha_{1}+\alpha_{2}\right)^{2}, \quad \alpha_{2}^{2}, \quad 0\right)$.

Applying the elimination procedure we get

$$
\iota_{[1,2]}\left(\left(Z_{[2]}^{T}\right)^{2}-\alpha_{2} Z_{[2]}^{T}\right)=3 \alpha_{1}\left(\alpha_{1}+\alpha_{2}\right),
$$

but

$$
\iota_{[1,2]}\left(Z_{[1,2]}^{T}\right)=\alpha_{1}\left(\alpha_{1}+\alpha_{2}\right),
$$

so

$$
\left(Z_{[2]}^{T}\right)^{2}=\alpha_{2} Z_{[2]}^{T}+3 Z_{[1,2]}^{T}
$$

and, in particular, $Z_{[2]}^{2}=3 Z_{[1,2]}$.

Continuing this way we can recover the whole multiplication table in $\mathrm{CH}_{T}^{*}\left(G_{0} / P\right)$ and $\mathrm{CH}^{*}\left(G_{0} / P\right)$.

Let us compute $S^{1}\left(Z_{[1,2,1,2]}^{T}\right)$ for $p=2$ now. The generalized Billey formula (5.1) gives

$$
\begin{aligned}
& \iota\left(Z_{[1,2,1,2]}^{T}\right)=\left(\left(\alpha_{1}+\alpha_{2}\right)\left(3 \alpha_{1}+2 \alpha_{2}\right)\left(2 \alpha_{1}+\alpha_{2}\right)\left(3 \alpha_{1}+\alpha_{2}\right),\right. \\
& \left.\alpha_{1}\left(3 \alpha_{1}+2 \alpha_{2}\right)\left(2 \alpha_{1}+\alpha_{2}\right)\left(3 \alpha_{1}+\alpha_{2}\right), \quad 0, \quad 0, \quad 0, \quad 0\right) \\
& =\left(\left(\alpha_{1}^{2}+\alpha_{2}^{2}\right) \alpha_{1} \alpha_{2}, \quad \alpha_{1}^{2} \alpha_{2}\left(\alpha_{1}+\alpha_{2}\right), \quad 0, \quad 0, \quad 0, \quad 0\right) \text {. }
\end{aligned}
$$

Substituting $\alpha_{1} \mapsto \alpha_{1}+t \alpha_{1}^{2}, \alpha_{2} \mapsto \alpha_{2}+t \alpha_{2}^{2}$, taking modulo 2 and taking the coefficient at $t$ we get:

$$
S^{1}\left(\iota\left(Z_{[1,2,1,2]}^{T}\right)\right)=\left(\left(\alpha_{1}^{2}+\alpha_{2}^{2}\right)\left(\alpha_{1}^{2} \alpha_{2}+\alpha_{1} \alpha_{2}^{2}\right), \quad \alpha_{1}^{3} \alpha_{2}\left(\alpha_{1}+\alpha_{2}\right), \quad 0, \quad 0, \quad 0, \quad 0\right)
$$

and the elimination procedure gives

$$
S^{1}\left(Z_{[1,2,1,2]}^{T}\right)=\alpha_{1} Z_{[1,2,1,2]}^{T}+Z_{[2,1,2,1,2]}^{T} \cdot
$$

In particular, $S^{1}\left(Z_{[1,2,1,2]}\right)=Z_{[2,1,2,1,2]}=$ pt. 
Now we compute the second Chern class $c_{2}^{T}$ of the tangent bundle of $G_{0} / P$. The roots of this bundle are:

$$
\alpha_{2}, \quad \alpha_{1}+\alpha_{2}, \quad 3 \alpha_{1}+2 \alpha_{2}, \quad 2 \alpha_{1}+\alpha_{2}, \quad 3 \alpha_{1}+\alpha_{2} .
$$

The total Chern class equals

$$
\left(1+t \alpha_{2}\right)\left(1+t\left(\alpha_{1}+\alpha_{2}\right)\right)\left(1+t\left(3 \alpha_{1}+2 \alpha_{2}\right)\right)\left(1+t\left(2 \alpha_{1}+\alpha_{2}\right)\right)\left(1+t\left(3 \alpha_{1}+\alpha_{2}\right)\right) .
$$

The coefficient at $t^{2}$ is $29 \alpha_{1}^{2}+14 \alpha_{2}^{2}+42 \alpha_{1} \alpha_{2}$. Now

$$
\begin{aligned}
& s_{2}\left(29 \alpha_{1}^{2}+14 \alpha_{2}^{2}+42 \alpha_{1} \alpha_{2}\right)=29 \alpha_{1}^{2}+\alpha_{2}^{2}+16 \alpha_{1} \alpha_{2}, \\
& s_{1}\left(29 \alpha_{1}^{2}+\alpha_{2}^{2}+16 \alpha_{1} \alpha_{2}\right)=-10 \alpha_{1}^{2}+\alpha_{2}^{2}-10 \alpha_{1} \alpha_{2}, \\
& s_{2}\left(-10 \alpha_{1}^{2}+\alpha_{2}^{2}-10 \alpha_{1} \alpha_{2}\right)=-10 \alpha_{1}^{2}+\alpha_{2}^{2}-10 \alpha_{1} \alpha_{2}, \\
& s_{1}\left(-10 \alpha_{1}^{2}+\alpha_{2}^{2}-10 \alpha_{1} \alpha_{2}\right)=29 \alpha_{1}^{2}+\alpha_{2}^{2}+16 \alpha_{1} \alpha_{2}, \\
& s_{2}\left(29 \alpha_{1}^{2}+\alpha_{2}^{2}+16 \alpha_{1} \alpha_{2}\right)=29 \alpha_{1}^{2}+14 \alpha_{2}^{2}+42 \alpha_{1} \alpha_{2},
\end{aligned}
$$

so we have

$$
\begin{aligned}
& \iota\left(c_{2}^{T}\right)=\left(29 \alpha_{1}^{2}+14 \alpha_{2}^{2}+42 \alpha_{1} \alpha_{2}, \quad 29 \alpha_{1}^{2}+\alpha_{2}^{2}+16 \alpha_{1} \alpha_{2}, \quad-10 \alpha_{1}^{2}+\alpha_{2}^{2}-10 \alpha_{1} \alpha_{2},\right. \\
& \left.-10 \alpha_{1}^{2}+\alpha_{2}^{2}-10 \alpha_{1} \alpha_{2}, \quad 29 \alpha_{1}^{2}+\alpha_{2}^{2}+16 \alpha_{1} \alpha_{2}, \quad 29 \alpha_{1}^{2}+14 \alpha_{2}^{2}+42 \alpha_{1} \alpha_{2}\right) .
\end{aligned}
$$

By the elimination procedure we obtain:

$$
c_{2}^{T}=\left(29 \alpha_{1}^{2}+14 \alpha_{2}^{2}+42 \alpha_{1} \alpha_{2}\right) Z_{[]}^{T}-13\left(2 \alpha_{1}+\alpha_{2}\right) Z_{[2]}^{T}+13 Z_{[1,2]}^{T} .
$$

In particular, the ordinary second Chern class of the bundle equals $13 Z_{[1,2]}$.

\section{Chernousov-Merkurjev formula}

Recall that $G$ denotes a semisimple algebraic group of inner type. Let $X$ and $X^{\prime}$ be twisted $G$-homogeneous flag varieties. We present $G$ as a twisted form of a split group $G_{0}$. Then $X$ and $X^{\prime}$ are twisted forms of $G_{0} / P$ and $G_{0} / P^{\prime}$ resp. for some standard parabolic subgroups $P, P^{\prime}$ of $G_{0}$. We say that $X$ and $X^{\prime}$ are homogeneous varieties of type $P$ and $P^{\prime}$ respectively.

In [CM, Proposition 13] Chernousov and Merkurjev construct a filtration by closed subvarieties on $X \times X^{\prime}$ such that the successive differences are affine fibrations over $Y_{w}$ of rank $l\left(W_{P} w W_{P^{\prime}}\right)$, where $w$ runs over the representatives of $W_{P} \backslash W / W_{P^{\prime}}$, $W, W_{P}, W_{P^{\prime}}$ are the Weyl groups of $G_{0}, P, P^{\prime}$ resp., $l\left(W_{P} w W_{P^{\prime}}\right)$ is the length of the minimal representative of the double coset $W_{P} w W_{P^{\prime}}$, and $Y_{w}$ is a twisted form of $G_{0} / Q_{w}$ with $Q_{w}=R_{u} P \cdot\left(P \cap w P^{\prime} w^{-1}\right)$, where $R_{u} P$ stands for the unipotent radical of $P$. Note that by [CM, Lemma 7] $Q_{w}$ is a standard parabolic subgroup of $G_{0}$ and is contained in $P$.

By [NeZ, Theorem 4.4] we get:

\subsection{Proposition. In the above notation}

$$
\mathrm{CH}^{*}\left(X \times X^{\prime}\right) \simeq \bigoplus_{w \in W_{P} \backslash W / W_{P^{\prime}}} \mathrm{CH}^{*-\operatorname{dim} X-\operatorname{dim} X^{\prime}+\operatorname{dim} Y_{w}+l\left(W_{P} w W_{P^{\prime}}\right)}\left(Y_{w}\right) .
$$

6.2. Remark. Theorem 4.4 in [NeZ] is proved for any oriented cohomology theory using resolution of singularities. So, it is assumed there that char $k=0$. For Chow groups this is not necessary, cf. [EKM, Theorem 66.2]. 
6.3. Example. If $G$ is a special orthogonal group, and $X=X^{\prime}=X_{1}$ is a projective quadric of dimension at least 5 , then

$$
\mathrm{CH}^{*}(X \times X) \simeq \mathrm{CH}^{*}(X) \oplus \mathrm{CH}^{*-1}\left(X_{1,2}\right) \oplus \mathrm{CH}^{*-\operatorname{dim} X}(X) .
$$

We now develop an important tool to produce rational projectors.

Let $w \in W_{P} \backslash W / W_{P^{\prime}}$ and $f: \bar{Y}_{w} \rightarrow \bar{X}$ be the natural map induced by the inclusion $Q_{w} \subset P$.

For a Schubert cycle $\beta=\left[\overline{B u Q_{w} / Q_{w}}\right] \in \mathrm{CH}^{*}\left(\bar{Y}_{w}\right), u \in W$, define an element $\beta_{\star}(1) \in \mathrm{CH}^{*}\left(\overline{X^{\prime}}\right)$ as

$$
\beta_{\star}(1)= \begin{cases}{\left[\overline{B u w P^{\prime} / P^{\prime}}\right],} & \text { if } l\left(u w W_{P^{\prime}}\right)=l\left(u W_{Q_{w}}\right)+l\left(W_{P} w W_{P^{\prime}}\right) ; \\ 0, & \text { otherwise, }\end{cases}
$$

and for an arbitrary $\beta \in \mathrm{CH}^{*}\left(\bar{Y}_{w}\right)$ define $\beta_{\star}(1)$ by linearity.

Fix a rational cycle $\alpha \in \mathrm{CH}^{*}\left(\bar{Y}_{w}\right)$ and for a cycle $x \in \mathrm{CH}^{*}(\bar{X})$ define

$$
\alpha_{\star}(x)=\left(\alpha \cdot f^{*}(x)\right)_{\star}(1) \in \mathrm{CH}^{*}\left(\overline{X^{\prime}}\right) .
$$

6.4. Theorem. In the preceding notation, for $\alpha \in \mathrm{CH}^{*}\left(\bar{Y}_{w}\right)$

$$
\alpha_{\star}: \mathrm{CH}(\bar{X}) \rightarrow \mathrm{CH}\left(\overline{X^{\prime}}\right)
$$

is the realization of a rational cycle on $\bar{X} \times \overline{X^{\prime}}$. Moreover, the realization of any rational cycle on $\bar{X} \times \overline{X^{\prime}}$ can be constructed in this way.

In particular, if $P=P^{\prime}$, then for $\alpha \in \mathrm{Ch}^{\operatorname{dim} Y_{w}-\operatorname{dim} X+l\left(W_{P} w W_{P}\right)}\left(\bar{Y}_{w}\right)$ some power of $\alpha_{\star}$ is the realization of a rational projector on $\bar{X}$, and the realization of any rational projector on $\bar{X}$ can be constructed in this way.

Proof. Let $X$ and $X^{\prime}$ be homogeneous $G$-varieties of type $P$ and $P^{\prime}$. Consider the following diagram

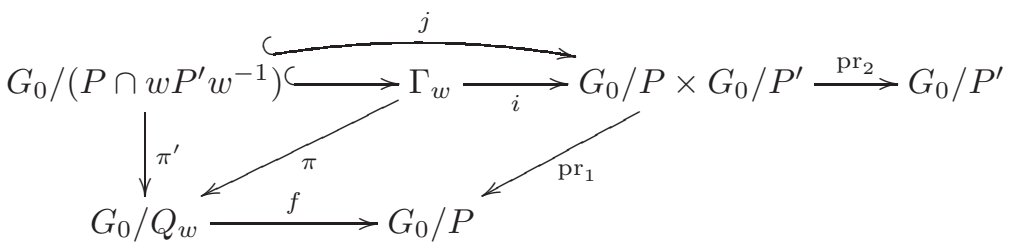

where the maps $\pi^{\prime}$ and $f$ are induced by inclusions $P \cap w P^{\prime} w^{-1} \subset Q_{w} \subset P$, $G_{0} /\left(P \cap w P^{\prime} w^{-1}\right)$ is considered as a subvariety of $G_{0} / P \times G_{0} / P^{\prime}$ under the map

$$
j: g\left(P \cap w P^{\prime} w^{-1}\right) \mapsto\left(g P, g w P^{\prime}\right), \quad g \in G_{0},
$$

$\Gamma_{w}$ is the closure in $G_{0} / P \times G_{0} / P^{\prime} \times G_{0} / Q_{w}$ of the image of the graph of $\pi^{\prime}$ under the map $j \times \mathrm{id}$, and $i$ and $\pi$ are induced by the projections.

The proof in [NeZ] shows that the image of an element $\alpha \in \mathrm{CH}^{*}\left(G_{0} / Q_{w}\right)$ under the isomorphism of Proposition 6.1 equals $i_{*} \pi^{*}(\alpha)$. Further, we identify the image of $\alpha$ with its realization, i.e., with the homomorphism

$$
\begin{aligned}
\alpha_{\star}: \mathrm{CH}^{*}\left(G_{0} / P\right) & \rightarrow \mathrm{CH}^{*}\left(G_{0} / P^{\prime}\right) \\
x & \mapsto\left(\operatorname{pr}_{2}\right)_{*}\left(i_{*} \pi^{*}(\alpha) \cdot \operatorname{pr}_{1}^{*}(x)\right) .
\end{aligned}
$$

The above diagram and the projection formula show that

$$
\begin{aligned}
\alpha_{\star}(x) & =\left(\operatorname{pr}_{2}\right)_{*}\left(i_{*} \pi^{*}(\alpha) \cdot \operatorname{pr}_{1}^{*}(x)\right)=\left(\operatorname{pr}_{2}\right)_{*}\left(i_{*}\left(\pi^{*}(\alpha) \cdot i^{*} \operatorname{pr}_{1}^{*}(x)\right)\right) \\
& =\left(\operatorname{pr}_{2}\right)_{*}\left(i_{*} \pi^{*}\left(\alpha \cdot f^{*}(x)\right)\right)=\left(\alpha \cdot f^{*}(x)\right)_{\star}(1) .
\end{aligned}
$$


In particular, to compute $\alpha_{\star}(x)$, we just need to know the image of $\beta_{\star}(1)$ for each element $\beta \in \mathrm{CH}^{*}\left(G_{0} / Q_{w}\right)$. One sees directly that for a Schubert cycle $\beta=$ $\left[\overline{B u Q_{w} / Q_{w}}\right]$

$$
\beta_{\star}(1)= \begin{cases}{\left[\overline{B u w P^{\prime} / P^{\prime}}\right],} & \text { if } l\left(u w W_{P^{\prime}}\right)=l\left(u W_{Q_{w}}\right)+l\left(W_{P} w W_{P^{\prime}}\right) ; \\ 0, & \text { otherwise. }\end{cases}
$$

To finish the proof of the theorem it remains to set $P^{\prime}=P$ and note that in $\operatorname{End}(\mathscr{M}(\bar{X}))$ some power of any element is a projector.

6.5. Example. Let $A$ be a central simple algebra of degree $n+1, G=\mathrm{PGL}_{1}(A)$, $P=P_{1}$, and $P^{\prime}=P_{n}$. Then $G_{0}=\mathrm{PGL}_{n+1}, X$ is the Severi-Brauer variety $\mathrm{SB}(A), X^{\prime}=\mathrm{SB}\left(A^{\text {op }}\right), W=\operatorname{Sym}(\{1, \ldots, n+1\})$ is the symmetric group on letters $1, \ldots, n+1, W_{P}=\operatorname{Sym}(\{2, \ldots, n+1\})$, and $W_{P^{\prime}}=\operatorname{Sym}(\{1, \ldots, n\})$.

The minimal representatives of the cosets $W / W_{P^{\prime}}$ are

$$
\left\{1, s_{n}, s_{n-1} s_{n}, \ldots, s_{1} \cdots s_{n-1} s_{n}\right\}
$$

where $s_{i}=(i, i+1)$ is a simple transposition, and the minimal representatives of the cosets $W / W_{P}$ are

$$
\left\{1, s_{1}, s_{2} s_{1}, \ldots, s_{n} \cdots s_{2} s_{1}\right\} .
$$

The minimal representatives of the double cosets $W_{P} \backslash W / W_{P^{\prime}}$ are $\left\{1, s_{1} \cdots s_{n-1} s_{n}\right\}$. Take $w=1$. Then $Q_{w}=P_{1, n}$.

By Proposition 6.1 we have

$$
\mathrm{CH}^{*}\left(\mathrm{SB}(A) \times \mathrm{SB}\left(A^{\mathrm{op}}\right)\right) \simeq \mathrm{CH}^{*-1}\left(\mathrm{SB}_{1, n}(A)\right) \oplus \mathrm{CH}^{*}(\mathrm{SB}(A)),
$$

where $\mathrm{SB}_{1, n}(A)$ is the variety of parabolic subgroups of type $P_{1, n}$ (known also as the incidence variety).

Take (a rational) $\alpha=1 \in \mathrm{CH}^{0}\left(G_{0} / P_{1, n}\right)$. Let $h_{1}$ and $h_{n}$ be the Schubert cycles in $\mathrm{CH}^{1}\left(G_{0} / P\right)$ and $\mathrm{CH}^{1}\left(G_{0} / P^{\prime}\right)$. All Schubert cycles in $\mathrm{CH}^{*}\left(G_{0} / P\right)$ equal $1, h_{1}, \ldots, h_{1}^{n}$. We compute $\alpha_{\star}\left(h_{1}^{i}\right)$ now.

We have $\alpha_{\star}\left(h_{1}^{i}\right)=\left(f^{*}\left(h_{1}^{i}\right)\right)_{\star}(1)$. The cycle $h_{1}^{i}$ equals $\left[\overline{B v_{i} P / P}\right]$ with $v_{i}=s_{n-i} \cdots s_{1}$ and $f^{*}\left(h_{1}^{i}\right)=\left[\overline{B v_{i} v_{0} P_{1, n} / P_{1, n}}\right]$ with $v_{0}=s_{2} \cdots s_{n}$. By formula $(6.2)$

$$
\left(f^{*}\left(h_{1}^{i}\right)\right)_{\star}(1)= \begin{cases}h_{n}, & \text { if } i=n ; \\ 1, & \text { if } i=n-1 ; \\ 0, & \text { otherwise. }\end{cases}
$$

Thus $\alpha$ as an element in $\mathrm{CH}^{*}\left(G_{0} / P \times G_{0} / P^{\prime}\right)$ equals $h_{1} \times 1+1 \times h_{n}$. So, the latter cycle is rational.

\section{WEAK SPECIAL CORRESPONDENCES}

7.1. Definition. Let $p$ be a prime number, and $X$ be a smooth projective irreducible variety over $k$ of dimension $b(p-1)$ for some $b$. A cycle $\rho \in \mathrm{Ch}^{b}(X \times X)$ is called a weak special correspondence, if $\rho_{k(X)}=H \times 1-1 \times H$ for some $H \in \mathrm{Ch}^{b}\left(X_{k(X)}\right)$, $\bar{\pi}:=c \cdot \rho_{k(X)}^{p-1}$ is a projector for some $c \in \mathbb{F}_{p}^{\times}$, and

$$
\left(X_{k(X)}, \bar{\pi}\right) \simeq \bigoplus_{i=0}^{p-1} \mathbb{F}_{p}(b i)
$$


7.2. Lemma (Rost, [Ro07, Section 9]). Assume that $X$ possesses a weak special correspondence, has no zero-cycles of degree coprime to $p$, and char $k=0$. Then $\operatorname{dim} X=p^{n}-1$ for some $n$.

7.3. Lemma. Assume that $p \in\{2,3\}$. Let $X$ be a smooth projective irreducible variety over $k$ of dimension $b(p-1)$ with no zero-cycles of degree coprime to $p$, and $\pi$ a projector over $k$ such that $\left(X_{k(X)}, \pi_{k(X)}\right) \simeq \bigoplus_{i=0}^{p-1} \mathbb{F}_{p}(b i)$. Then $X$ possesses a weak special correspondence.

Proof. Denote $\bar{\pi}=\pi_{k(X)}$ and $\bar{X}=X_{k(X)}$. Since $(\bar{X}, \bar{\pi}) \simeq \bigoplus_{i=0}^{p-1} \mathbb{F}_{p}(b i)$, the projector $\bar{\pi}$ equals $\sum_{i=0}^{p-1} h_{i} \times g_{i}$ for some $h_{i} \in \mathrm{Ch}^{b i}(\bar{X})$ and $g_{i} \in \mathrm{Ch}_{b i}(\bar{X})$ with $\operatorname{deg}\left(h_{i} g_{i}\right)=1$ for all $i$.

Note first that $\pi^{t} \circ \pi$ contains at most $p$ summands and is non-zero, since

$$
\left(g_{p-1} \times h_{p-1}\right) \circ\left(h_{0} \times g_{0}\right)=d \cdot h_{0} \times h_{p-1} \neq 0,
$$

where $d=\operatorname{deg}\left(g_{0} g_{p-1}\right) \in \mathbb{F}_{p}^{\times}$. Therefore, since $X$ has no zero-cycles of degree coprime to $p$, we can assume that $g_{i}=h_{p-1-i}$ for all $i$. In particular, this proves our lemma for $p=2$.

Write $f: \operatorname{Spec} k(X) \rightarrow X$ for the generic point. By the generic point diagram (see [PSZ, Lemma 1.8]) there is a cycle $\alpha \in \mathrm{Ch}^{b}(\bar{X} \times \bar{X})$ such that $\beta:=h_{1} \times 1+\alpha$ is defined over $k$ and $\left(\mathrm{id}_{X}^{*} \times f\right)(\alpha)=0$.

Consider $\bar{\pi} \circ \beta \circ \bar{\pi}$. A direct computation shows that this cycle equals $\rho_{1}:=$ $h_{1} \times 1+a_{1} 1 \times h_{1}$ for some $a_{1} \in \mathbb{F}_{p}$. By symmetry we can assume that $a_{1} \neq 0$. If $p=3$, then set $c=\operatorname{deg}\left(h_{1}^{2}\right)^{-1} \in \mathbb{F}_{p}^{\times}$. The cycle $\rho_{1}^{2}=h_{1}^{2} \times 1-a_{1} h_{1} \times h_{1}+1 \times h_{1}^{2}$. Since $X$ has no zero-cycles of degree coprime to $p$, we have $a_{1}=-1$. Moreover, $c \cdot \rho_{1}^{2}$ is a projector. Thus, $\rho_{1}$ is a weak special correspondence on $X$.

7.4. Remark. Using messier computations one can also prove the above Lemma for $p=5$.

7.5. Lemma. Let $X$ be a smooth projective irreducible variety over $k$ with $\operatorname{char} k=0$ and $M$ a direct summand of its motive. Assume that $M$ is indecomposable and generically split and $M_{k(X)} \simeq \bigoplus_{i \in I \cup\{0\}} \mathbb{F}_{p}(i)$ for some multiset of positive indexes $I$.

Then there exists a smooth projective irreducible variety $Y$ over $k$ such that $M$ is isomorphic to an upper direct summand of $\mathscr{M}(Y)$ and $\operatorname{dim} M=\operatorname{dim} Y$.

Proof. Let $Y^{\prime}$ be a closed irreducible subvariety of $X$ of minimal dimension with respect to the property that $Y_{k(X)}^{\prime}$ has a zero-cycle of degree coprime to $p$.

By [Sem, Lemma 7.1] there exists a smooth projective irreducible variety $\widetilde{Y^{\prime}}$ birational to $Y^{\prime}$ such that both $\widetilde{Y^{\prime}}{ }_{k(X)}$ and $X_{k\left(\widetilde{Y^{\prime}}\right)}$ have zero-cycles of degree coprime to $p$. Since the upper motive $M$ of $X$ is generically split, Rost nilpotence holds for its endomorphism ring, i.e., the kernel of the natural map $\operatorname{End}(M) \rightarrow \operatorname{End}(\bar{M})$ consists of nilpotent correspondences by [ViZ, Prop. 3.1]. Therefore $M$ is also an upper direct summand of $\widetilde{Y^{\prime}}$. Hence, $\operatorname{dim} Y^{\prime}=\operatorname{dim} \widetilde{Y}^{\prime} \geq \operatorname{dim} M$.

Let now $Y^{\prime \prime}$ be the generic point of $M$ (see Lemma 3.5). Obviously, $Y_{k(X)}^{\prime \prime}$ in not 0 in $\operatorname{Ch}\left(X_{k(X)}\right)$, and therefore without loss of generality we can assume that $Y^{\prime \prime}$ is represented by a closed subvariety of $X$, which we denote by the same letter. By [KaM, Remark 5.6] the variety $Y^{\prime \prime}$ has the property that $Y_{k(X)}^{\prime \prime}$ has a zero-cycle of 
degree coprime to $p$. Since $\operatorname{dim} Y^{\prime \prime}=\operatorname{dim} M$, the dimension of $Y^{\prime \prime}$ is minimal with respect to this property.

Therefore by [Sem, Lemma 7.1] there exists a smooth projective irreducible variety $Y$ birational to $Y^{\prime \prime}$ with required properties.

The following statement for $p=2$ might be called a binary motive theorem.

7.6. Corollary. Assume that $p \in\{2,3\}$ and char $k=0$. Let $X$ be a smooth projective irreducible variety with no zero-cycles of degree coprime to $p$ and $M$ a direct summand of $\mathscr{M}(X)$. If $M_{k(X)} \simeq \bigoplus_{i=0}^{p-1} \mathbb{F}_{p}($ bi) for some integer $b$, then $\operatorname{dim} M=p^{n}-1$ for some $n$.

7.7. Proposition. Let $G$ be a split semisimple algebraic group of inner type over a field $k$ with char $k=0$ and $\xi \in H^{1}(k, G)$. Let $p \in\{2,3\}$. Consider a twisted ${ }_{\xi} G$-homogeneous flag variety $X$ and write

$$
\mathscr{M}\left(X_{k(X)}\right) \simeq \oplus_{i \in I} \mathbb{F}_{p}(i) \bigoplus \oplus_{j \in J} N_{j}
$$

with indecomposable direct summands $N_{j}$ of positive dimension.

Assume that the following conditions hold:

(1) For all $j$ the motives $N_{j}$ are defined over $k$. Moreover, there exist twisted flag varieties $Y_{j}$ over $k$ such that $N_{j}=\mathcal{U}\left(Y_{j}\right)$ and every cycle in $\operatorname{Ch}\left(\overline{Y_{j} \times X}\right)$ which is defined over $k\left(Y_{j}\right)\left(X_{k\left(Y_{j}\right)}\right)$ is defined over $k\left(Y_{j}\right)$.

(2) The variety $X$ has no zero-cycles of degree coprime to $p$.

(3) Let $Q(t)$ denote the Poincaré polynomial of the (graded by codimension) subgroup of $\mathrm{Ch}^{*}(\bar{X})$ generated by the rational cycles of the first shell. Assume

$$
\frac{\sum_{i \in I} t^{i}}{Q(t)}=\sum_{l=0}^{p-1} t^{b l}
$$

for some $b$.

Then $b=\frac{p^{n}-1}{p-1}$ for some integer $n$.

Proof. Since by assumption the motives $N_{j}$ are defined over $k$, we use for simplicity the same notation $N_{j}$ over $k$ and over $k(X)$.

Since $N_{j}$ are defined over $k$, are indecomposable over $k(X)$ and have positive dimension, we can apply Proposition 3.2. So,

$$
\mathscr{M}(X) \simeq U \oplus \bigoplus_{j \in J} N_{j}
$$

over $k$, where $U$ is a motive with Poincaré polynomial $\sum_{i \in I} t^{i}$, since by our assumptions $U_{k(X)} \simeq \oplus_{i \in I} \mathbb{F}_{p}(i)$.

It follows from Theorem 4.10 that $U \simeq \oplus_{s \in S} M(s)$ for some motive $M$ and $Q(t)=\sum_{s \in S} t^{s}$. In particular, by assumption (3), $P(M, t)=\sum_{l=0}^{p-1} t^{b l}$. The proposition follows now from Corollary 7.6.

\section{Applications to motives of tWisted flag Varieties: type $\mathrm{E}_{6}$}

The goal of this section is to provide a complete classification of all possible motivic decompositions of twisted $G$-homogeneous flag varieties for $G$ a group of inner type $\mathrm{E}_{6}$. Note that with $\mathbb{F}_{p}$-coefficients and $p \neq 2,3$, every twisted $G$-homogeneous flag variety is a direct sum of Tate motives, and the case $p=2$ was settled in [PSZ, 
p. 1048]. Therefore we only consider $\mathbb{F}_{3}$-coefficients here. All decomposition types are collected in Table 8A.

Throughout we will refer to the Tits algebra $A$ of $G$, by which we mean a Tits algebra for the vertex 1 in the sense of [Ti71, 6.4.1]. (This is a special case of the more general theory of Tits algebras from [Ti71] or [KMRT, §27].) This algebra $A$ is a central simple algebra of degree 27 and is determined up to isomorphism or anti-isomorphism by $G$. By $D$ we denote the underlying central simple division algebra.

\begin{tabular}{|c|c|c|}
\hline$J_{3}(G)$ & $\Theta$ & $\mathscr{M}\left(X_{\Theta}\right)$ \\
\hline \multirow[t]{4}{*}{$(2,1)$} & 2 & $M_{2,1} \oplus M_{2,1}(1)$ \\
\hline & & $M_{2,1} \oplus\left(\oplus_{j \in J^{2,1}} R_{2,1}(j)\right) \oplus M_{2,1}(9)$ \\
\hline & $\{2,4\}$ & $\mathscr{M}\left(X_{4}\right) \oplus \mathscr{M}\left(X_{4}\right)(1)$ \\
\hline & any other & $\bigoplus_{i \in I_{\odot}^{2,1}} R_{2,1}(i)$ \\
\hline \multirow[t]{4}{*}{$(1,1)$} & 2 & $M_{1,1} \oplus\left(\oplus_{i=4}^{7} R_{1,1}(i)\right) \oplus M_{1,1}(1)$ \\
\hline & & $M_{1,1} \oplus\left(\oplus_{j \in J^{1,1}} R_{1,1}(j)\right) \oplus M_{1,1}(9)$ \\
\hline & $\{2,4\}$ & $\mathscr{M}\left(X_{4}\right) \oplus \mathscr{M}\left(X_{4}\right)(1)$ \\
\hline & any other & $\bigoplus_{i \in I_{\Theta}^{1,1}} R_{1,1}(i)$ \\
\hline$(0,1)$ & any & $\bigoplus_{i \in I_{\Theta}^{0,1}} R_{0,1}(i)$ \\
\hline \multirow[t]{4}{*}{$(1,0)$} & 2 & $\bigoplus_{i=0,1,10,11,20,21} \mathbb{F}_{3}(i) \oplus \bigoplus_{j \in J_{2}^{1,0}} \mathscr{M}(\mathrm{SB}(D))(j)$ \\
\hline & & $\bigoplus_{i=0,1,9,10,10,11,19,20,20,21,29,30} \mathbb{F}_{3}(i) \oplus \bigoplus_{j \in J_{4}^{1,0}} \mathscr{M}(\mathrm{SB}(D))(j)$ \\
\hline & $\{2,4\}$ & $\mathscr{M}\left(X_{4}\right) \oplus \mathscr{M}\left(X_{4}\right)(1)$ \\
\hline & any other & $\bigoplus_{i \in I_{\Theta}^{1,0}} \mathscr{M}(\mathrm{SB}(D))(i)$ \\
\hline$(0,0)$ & any & $\oplus_{i \in I^{0,0}, \mathbb{F}_{3}(i)}$ \\
\hline
\end{tabular}

TABle 8A. Motivic decomposition of twisted flag varieties of $\mathrm{E}_{6}$ $\bmod 3$

Motive $\mid$ Poincaré polynomial

\begin{tabular}{l|l}
$M_{2,1}$ & $\frac{\left(t^{4}+1\right)\left(t^{12}-1\right)\left(t^{6}+t^{3}+1\right)}{t^{2}-1}$ \\
$M_{1,1}$ & $t^{20}+t^{18}+t^{17}+t^{16}+t^{14}+t^{13}+t^{12}+t^{11}+2 t^{10}+t^{9}+t^{8}+t^{7}+t^{6}+t^{4}+t^{3}+t^{2}+1$ \\
$R_{j_{1}, j_{2}}$ & $\frac{\left(t^{3^{j}}-1\right)\left(t^{4 \cdot 3} 3^{j_{2}}-1\right)}{(t-1)\left(t^{4}-1\right)}$
\end{tabular}

TABLE 8B. Poincaré polynomials of some motives from Table 8A

\begin{tabular}{c|l} 
Multiset of indexes & Polynomial \\
\hline$I_{\Theta}^{j_{1}, j_{2}}$ & $\frac{P\left(X_{\Theta}, t\right)}{P\left(R_{\left.j_{1}, j_{2}, t\right)}\right.}$ \\
$J^{j_{1}, 1}$ & $\frac{P\left(X_{4}, t\right)-P\left(M_{j_{1}, 1}, t\right)\left(1+t^{9}\right)}{P\left(R_{\left.j_{1}, 1, t\right)}, t\right)}$ \\
$J_{2}^{1,0}$ & $\frac{P\left(X_{2}, t\right)-\left(1+t+t^{10}+t^{11}+t^{20}+t^{21}\right)}{1+t+t^{2}}$ \\
$J_{4}^{1,0}$ & $\frac{P\left(X_{4}, t\right)-\left(1+t+t^{10}+t^{11}+t^{20}+t^{21}\right)\left(1+t^{9}\right)}{1+t+t^{2}}$
\end{tabular}

TABLE 8C. Multisets of indexes appearing in Table 8A 
Left column: the $J$-invariant. Let $G_{0}$ be a split semisimple algebraic group over $k$ and $p$ be a prime. Denote $\bar{G}=G_{0} \times_{k} k_{\text {sep }}$, where $k_{\text {sep }}$ is a separable closure of $k$. It is known that

$$
\mathrm{Ch}^{*}(\bar{G}) \simeq \mathbb{F}_{p}\left[x_{1}, \ldots, x_{r}\right] /\left(x_{1}^{p^{k_{1}}}, \ldots, x_{r}^{p^{k_{r}}}\right)
$$

with $\operatorname{deg} x_{i}=d_{i}$ for some integers $r, k_{i}$, and $d_{i}$. We order the generators so that $d_{1} \leq \ldots \leq d_{r}$ and fix one such isomorphism between $\mathrm{Ch}^{*}(\bar{G})$ and this polynomial ring.

Let now $\xi \in Z^{1}\left(k, G_{0}\right)$ be a cocycle and consider the composite map

$$
\mathrm{Ch}\left(\xi\left(G_{0} / B\right)\right) \stackrel{\text { res }}{\longrightarrow} \mathrm{Ch}\left(\xi\left(G_{0} / B\right) \times_{k} k_{\text {sep }}\right) \stackrel{\simeq}{\rightarrow} \mathrm{Ch}\left(G_{0} / B \times_{k} k_{\text {sep }}\right) \rightarrow \operatorname{Ch}(\bar{G}),
$$

where $B$ is a Borel subgroup of $G_{0}$ defined over $k$, the first map is the restriction map, the second map is induced by the isomorphism

$$
\xi\left(G_{0} / B\right) \times_{k} k_{\mathrm{sep}} \simeq G_{0} / B \times_{k} k_{\mathrm{sep}}
$$

given by $\xi$, and the third map is induced by the canonical quotient map. According to [PSZ, Definition 4.6] one can associate an invariant

$$
J_{p}(\xi)=\left(j_{1}, \ldots, j_{r}\right) \in \mathbb{Z}^{r}
$$

which measures the "size" of the image of this composite map. It does not depend on the choice of a separable closure $k_{\text {sep }}$.

Formally speaking, $J_{p}(\xi)$ is an invariant of $\xi$, not of $\xi\left(G_{0}\right)$. But if $G_{0}$ is simple and not of type $\mathrm{D}$ or $p \neq 2$, then the degrees $d_{i}$ are pairwise distinct, and it is a well-defined invariant of the twisted form $G={ }_{\xi}\left(G_{0}\right)$ and we denote this invariant by $J_{p}(G)$. For the excluded case where $G_{0}$ has type D and $p=2$, see [QSZ].

We remark that some constraints on the $J$-invariants are classified in [PSZ, Table 4.13]. E.g., if $G_{0}$ (equivalently, $G$ ) is adjoint of type $\mathrm{E}_{6}$ and $p=3$, then $r=2, d_{1}=1, d_{2}=4, k_{1}=2, k_{2}=1, j_{1} \in\{0,1,2\}$, and $j_{2} \in\{0,1\}$. We prove below that there are actually further constraints on the $J$-invariant, see e.g. Corollary 8.10.

Remaining columns. For the second column, recall that the simple roots of $\mathrm{E}_{6}$ are numbered as in the diagram

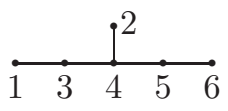

The motives $M_{j_{1}, j_{2}}$ and $R_{j_{1}, j_{2}}$ listed in the third column are indecomposable, and the latter is the upper motive of the variety of Borel subgroups. Their Poincaré polynomials are given in Table $8 \mathrm{~B}$. The multisets of indexes $I_{\Theta}^{j_{1}, j_{2}}$ and $J^{j_{1}, j_{2}}$ in Table 8A are defined as follows: an integer $i$ appears in the multiset $s$ times iff $s$ is the coefficient at $t^{i}$ of the respective polynomial given in Table 8C.

Each row of Table 8A occurs over a suitable field for a suitable group. The rest of this section and the next section are devoted to the proof of these tables.

By [PS10, Prop. 4.2] the Tits algebra $A$ is split iff the first slot $j_{1}$ in $J_{3}(G)$ equals 0 . If $j_{1}=0$, then every projective homogeneous $G$-variety is generically split over a field extension of degree coprime to 3 and this case was settled in [PSZ]. This immediately gives all rows of Table $8 \mathrm{~A}$ with $j_{1}=0$. 
Picard groups and Tits algebras. In this article we use some relations between rationality of the Picard groups of twisted flag varieties and their Tits algebras, see [MT95]. E.g., if all Tits algebras of a group $G$ of inner type are split algebras, then the Picard groups of all twisted flag varieties for the group $G$ are rational.

In this section, $G$ has inner type $\mathrm{E}_{6}$, and it follows from [MT95] that the Picard groups of varieties $X_{2}, X_{4}$, and $X_{2,4}$ are always rational.

We start now with some general observations.

8.1. Lemma. Let $\Delta$ be a Dynkin diagram (not necessarily of type $\mathrm{E}_{6}$ ) and $\Psi \subseteq \Theta \subseteq$ $\Delta$ two subsets of its vertices. Assume that $X_{\Theta}$ has a rational point over $k\left(X_{\Psi}\right)$, and

$$
P\left(X_{\Theta}, t\right) / P\left(X_{\Psi}, t\right)=t+1 .
$$

Then $\mathscr{M}\left(X_{\Theta}\right)=\mathscr{M}\left(X_{\Psi}\right) \oplus \mathscr{M}\left(X_{\Psi}\right)(1)$.

Proof. Since $\Psi \subseteq \Theta$, we have a natural map $f: X_{\Theta} \rightarrow X_{\Psi}$. The fibre $Z$ of $f$ over $k\left(X_{\Psi}\right)$ is a twisted flag variety over $k\left(X_{\Psi}\right)$. By the assumptions the Poincaré polynomial $P(Z, t)=P\left(X_{\Theta}, t\right) / P\left(X_{\Psi}, t\right)=t+1$, and $Z$ has a rational point. Therefore $Z$ is isomorphic to $\mathbb{P}^{1}$.

Now by [PSZ, Lemma 3.3] $f$ is a locally trivial fibration with fiber $\mathbb{P}^{1}$. Therefore [PSZ, Lemma 3.2] implies the claim.

This lemma with $\Psi=\{4\}$ and $\Theta=\{2,4\}$ and the classification of Tits indices immediately imply all rows of Table $8 \mathrm{~A}$ for $X_{2,4}$.

8.2. Lemma. If $X_{2}$ has a zero-cycle of degree coprime to 3, then $J_{3}(G)=(0,0)$ or $(1,0)$ and the index of $A$ is 1 or 3 respectively.

Proof. As $J_{3}(G)$ is unchanged if we replace $k$ with an extension of degree coprime to 3 [PSZ, Prop. 5.18(2)], we may assume that $X_{2}$ has a $k$-point. By the classification of Tits indexes, $G$ is split or has semisimple anisotropic kernel of type $2 \mathrm{~A}_{2}$.

In the second case ind $A=3$ and therefore $J_{3}\left(\mathrm{PGL}_{1}(A)\right)=(1)$. Thus, by [PS10, Prop. 3.9(2)] $J_{3}(G)=(1,0)$.

8.3. Lemma. The upper motives of $X_{2}$ and $X_{4}$ are isomorphic. If every zero-cycle on $X_{2}$ has degree divisible by 3 and the Tits algebra of $G$ is not split, then the dimension of its upper motive equals 20.

Proof. By hypothesis on $X_{2}, G$ is anisotropic and the Tits 3-indexes of $G_{K}$ as $K$ varies over all extensions $K$ of $k$ are empty, all of $\Delta$, and $\{2,4\}$. The claim on upper motives follows. Moreover, as in Example 4.7, there are (at most) two different big shells, the first shell $\mathrm{SH}_{\leqslant\{2\}}$ and the last shell $\mathrm{SH}_{\leqslant\{1\}}$.

As before write $\mathcal{U}\left(X_{2}\right)$ for the upper motive of $X_{2}$. An explicit computation of the decomposition of [CGM, Theorem 7.5] for $\mathscr{M}\left(X_{2}\right)$ shows that over $k\left(X_{2}\right)$ the motive of $X_{2}$ contains exactly six Tate motives: $\mathbb{F}_{3}, \mathbb{F}_{3}(1), \mathbb{F}_{3}(10), \mathbb{F}_{3}(11), \mathbb{F}_{3}(20)$, and $\mathbb{F}_{3}(21)$, and, by assumption, the variety $X_{2}$ does not have a zero-cycle of degree coprime to 3 . Therefore the number of Tate motives contained in $\mathcal{U}\left(X_{2}\right)$ over $k\left(X_{2}\right)$ is divisible by 3 .

Fix a generator $h$ of the Picard group of $X_{2}$; it is unique up to sign. This cycle is defined over $k$. Therefore, by Theorem 4.10 the motive $\mathcal{U}\left(X_{2}\right)(1)$ is a direct summand of $\mathscr{M}\left(X_{2}\right)$. All this implies that $\operatorname{dim} \mathcal{U}\left(X_{2}\right)=20$. 
8.4. Lemma. Let $J_{3}(G)=\left(j_{1}, j_{2}\right)$ with $j_{1} \neq 0$ and $M_{j_{1}, j_{2}}$ denote the upper motive of $X_{2}$. If $J_{3}(G) \neq(1,0)$, then

$$
\begin{aligned}
& \mathscr{M}\left(X_{2}\right) \simeq M_{j_{1}, j_{2}} \oplus M_{j_{1}, j_{2}}(1) \oplus \bigoplus_{i \in I_{1}} R_{j_{1}, j_{2}}(i) \quad \text { and } \\
& \mathscr{M}\left(X_{4}\right) \simeq M_{j_{1}, j_{2}} \oplus M_{j_{1}, j_{2}}(9) \oplus \bigoplus_{i \in I_{2}} R_{j_{1}, j_{2}}(i)
\end{aligned}
$$

for some multisets of indexes $I_{1}$ and $I_{2}$ (depending on $j_{1}, j_{2}$ ).

Proof. The formula for $X_{2}$ immediately follows from the proof of Lemma 8.3 and from Karpenko's theorem.

Consider now $X_{4}$. An explicit computation of the decomposition of [CGM, Theorem 7.5] for $\mathscr{M}\left(X_{4}\right)$ shows that over $k\left(X_{4}\right)$ its motive contains exactly 6 Tate motives: $\mathbb{F}_{3}, \mathbb{F}_{3}(9), \mathbb{F}_{3}(10), \mathbb{F}_{3}(19), \mathbb{F}_{3}(20), \mathbb{F}_{3}(29)$. Since the upper motives of $X_{2}$ and $X_{4}$ are isomorphic, we get

$$
\mathscr{M}\left(X_{4}\right)=M_{j_{1}, j_{2}} \oplus M_{j_{1}, j_{2}}(9) \oplus \bigoplus_{i \in I_{2}} R_{j_{1}, j_{2}}(i)
$$

for some multiset of indexes $I_{2}$.

Note that

$$
\begin{aligned}
& P\left(\mathrm{E}_{6} / P_{2}, t\right)=\frac{\left(t^{4}+1\right)\left(t^{12}-1\right)\left(t^{6}+t^{3}+1\right)}{t-1} \text { and } \\
& P\left(\mathrm{E}_{6} / P_{4}, t\right)=\frac{\left(t^{5}-1\right)\left(t^{3}+1\right)\left(t^{8}-1\right)\left(t^{6}+t^{3}+1\right)\left(t^{12}-1\right)}{(t-1)\left(t^{2}-1\right)^{2}} .
\end{aligned}
$$

So, to finish the proof Tables $8 \mathrm{~A}-8 \mathrm{C}$, it suffices to compute the Poincaré polynomials of $M_{2,1}$ and $M_{1,1}$, to find motivic decompositions for $J_{3}(G)=(1,0)$, and to exclude the case $J_{3}(G)=(2,0)$.

8.5. Lemma. $P\left(M_{2,1}, t\right)=\frac{\left(t^{4}+1\right)\left(t^{12}-1\right)\left(t^{6}+t^{3}+1\right)}{t^{2}-1}$.

Proof. If $2 \in I_{1}$ (in the notation of Lemma 8.4), then by Theorem 4.10, $3 \in I_{1}$, since for any $\alpha \in \mathrm{Ch}^{2}\left(\bar{X}_{2}\right)$ one has $\alpha \cdot h \neq 0$. And if $3 \in I_{1}$, then $4 \in I_{1}$, since for any $\beta \in \mathrm{Ch}^{3}\left(\bar{X}_{2}\right)$ one has $\beta \cdot h \neq 0$.

Thus, if $I_{1}$ is non-empty, then it contains an index $\geq 4$. But the Poincaré polynomial of $R_{2,1}$ equals $\left(1+t^{4}+t^{8}\right)\left(t^{9}-1\right) /(t-1)$, in particular, has dimension 16.

But by Lemma 8.4 we have

$$
P\left(X_{2}, t\right)=(t+1) P\left(M_{2,1}, t\right)+t^{m} P\left(R_{2,1}, t\right)+Q(t)
$$

where $m \geq 4, \operatorname{deg} P\left(M_{2,1}, t\right)=20$ by Lemma 8.3 and the polynomial $Q(t) \in \mathbb{Z}[t]$ has non-negative coefficients. Comparing the terms gives a contradiction.

8.6. Lemma. Assume $J_{3}(G)=(1,1)$. Then there exists a direct summand of the motive of $X_{2}$ starting in codimension 4.

Proof. Let $X=X^{\prime}=X_{2}$. A direct computation of all parameters of Proposition 6.1 shows that

$$
\begin{array}{r}
\mathrm{CH}^{*}\left(\mathrm{E}_{6} / P_{2} \times \mathrm{E}_{6} / P_{2}\right) \simeq \mathrm{CH}^{*}\left(\mathrm{E}_{6} / P_{2}\right) \oplus \mathrm{CH}^{*-1}\left(\mathrm{E}_{6} / P_{2,4}\right) \oplus \mathrm{CH}^{*-6}\left(\mathrm{E}_{6} / P_{1,2,6}\right) \\
\oplus \mathrm{CH}^{*-11}\left(\mathrm{E}_{6} / P_{2,4}\right) \oplus \mathrm{CH}^{*-21}\left(\mathrm{E}_{6} / P_{2}\right),
\end{array}
$$

where $\mathrm{E}_{6}$ stands for the split group of type $\mathrm{E}_{6}$. 
Let $h_{i}$ denote the generator of $\mathrm{Ch}^{1}\left(\bar{X}_{i}\right)$. Since $J_{3}(G)=(1,1)$, by [PS10, Proposition 4.2] $h_{1}^{3}$ is rational. Consider the rational cycle $\alpha=h_{1}^{6} \cdot c_{9} \in \mathrm{Ch}^{15}\left(\mathrm{E}_{6} / P_{1,2,6}\right)$, where $c_{9}$ stands for the 9 -th Chern class of the tangent bundle to $\bar{X}_{1,2,6}$. Another direct computation using Section 5 and formulas (6.1) and (6.2) shows that the realization $\alpha_{\star}: \mathrm{Ch}^{*}(\bar{X}) \rightarrow \mathrm{Ch}^{*}(\bar{X})$ maps $\mathrm{Ch}^{i}(\bar{X})$ to zero for $i \leq 3$, and maps $h_{2}^{4}$ to $-h_{2}^{4}(\bmod 3)$. In particular, by Theorem $6.4 \alpha$ defines a projector with generic point of codimension 4 .

8.7. Lemma. $P\left(M_{1,1}, t\right)=t^{20}+t^{18}+t^{17}+t^{16}+t^{14}+t^{13}+t^{12}+t^{11}+2 t^{10}+t^{9}+t^{8}+t^{7}+t^{6}+t^{4}+t^{3}+t^{2}+1$.

Proof. If $2 \in I_{1}$ or $3 \in I_{1}$ (in the notation of Lemma 8.4), then the same argument as in the proof of Lemma 8.5 implies that $3,4,5,6,7 \in I_{1}$. We have:

$$
P\left(X_{2}, t\right)=P\left(M_{1,1}, t\right)(1+t)+P\left(R_{1,1}, t\right) Q_{1,1}(t)
$$

with $Q_{1,1}(t)=t^{3}+t^{4}+t^{5}+t^{6}+t^{7}+Q(t)$ and

$$
P\left(M_{1,1}, t\right)=1+t^{10}+t^{20}+P\left(R_{1,0}, t\right) S(t)
$$

for some polynomials $Q$ and $S$ with non-negative coefficients. Comparing the terms we come to a contradiction.

Thus, 2 and $3 \notin I_{1}$. By Lemma $8.64 \in I_{1}$. Therefore $5,6,7 \in I_{1}$.

Since $2,3 \notin I_{1}$, these codimensions belong to the upper motive $M_{1,1}$. Therefore $P\left(M_{1,1}, t\right)=1+t^{10}+t^{20}+t^{2}+t^{3}+Q_{1}$ for some $Q_{1} \in \mathbb{Z}[t]$ with non-negative coefficients. Since $P\left(M_{1,1}\right)-\left(1+t^{10}+t^{20}\right)$ is divisible by $1+t+t^{2}$, we have $Q_{1}=t^{4}+Q_{2}$ for some $Q_{2} \in \mathbb{Z}[t]$ with non-negative coefficients.

By symmetry of the projector, $Q_{2}=t^{18}+t^{17}+t^{16}+Q_{3}$ for some $Q_{3} \in \mathbb{Z}[t]$ with non-negative coefficients, and this together with above polynomial identities implies that $Q_{3}=Q_{4} \cdot t^{6}$ for some $Q_{4} \in \mathbb{Z}[t]$, and $\operatorname{deg} Q_{3}=15<\operatorname{dim} R_{1,1}+6=16$. Therefore $I_{1} \subset\{4,5,6,7\}$, and, thus, $I_{1}=\{4,5,6,7\}$.

In the following statements we assume that char $k=0$ so that me way apply Proposition 7.7. However, we will remove this restriction in Corollary 10.4.

8.8. Lemma. If $J_{3}(G)=(1,0)$ and char $k=0$, then $X_{2}$ has a zero-cycle of degree coprime to 3 , and in particular $M_{1,0} \simeq \mathbb{F}_{3}$.

Proof. Assume $X_{2}$ has no zero-cycles of degree coprime to 3. Let $A$ be the Tits algebra of $G$ and $D$ the underlying division algebra. Denote by $Y$ the Severi-Brauer variety $\mathrm{SB}(D)$ of $D$. Since $J_{3}(G)=(1,0)$, ind $A=3$ and by [PS10, Theorem 5.7(3)] the variety $X_{2}$ is not generically split, and, hence, $\operatorname{ind}\left(A_{k\left(X_{2}\right)}\right)=3$. Therefore the motive of $Y_{k\left(X_{2}\right)}$ is indecomposable [Ka95, Th. 2.2.1].

Moreover, over $k\left(X_{2}\right)$ the motive $\mathscr{M}\left(X_{2}\right)$ is isomorphic to

$$
\oplus_{i=0,1,10,11,20,21} \mathbb{F}_{3}(i) \oplus\left(\oplus_{j \in J} \mathscr{M}\left(Y_{k\left(X_{2}\right)}\right)(j)\right)
$$

for some multiset of indexes $J$ by [CGM, Theorem 7.5].

Pick a generator $h$ of the Picard group of $X_{2}$. The proof of Lemma 8.3 shows that this is a rational cycle from the first shell. Now all conditions of Proposition 7.7 are satisfied and the parameter $b$ in that proposition equals 10 . This is a contradiction, because $10 \neq \frac{3^{n}-1}{2}$ for any $n$. 
8.9. Corollary. If char $k=0$ and $J_{3}(G)=(1,0)$, then

$$
\begin{aligned}
& \mathscr{M}\left(X_{2}\right) \simeq \oplus_{i=0,1,10,11,20,21} \mathbb{F}_{3}(i) \oplus\left(\oplus_{j \in J_{2}^{1,0}} \mathscr{M}(\operatorname{SB}(D))(j)\right) \quad \text { and } \\
& \mathscr{M}\left(X_{4}\right) \simeq \oplus_{i=0,1,9,10,10,11,19,20,20,21,29,30} \mathbb{F}_{3}(i) \oplus\left(\oplus_{j \in J_{4}^{1,0}} \mathscr{M}(\operatorname{SB}(D))(j)\right) .
\end{aligned}
$$

8.10. Corollary. Assume that char $k=0$. Then $J_{3}(G) \neq(2,0)$.

Proof. Let $A$ be the Tits algebra of $G$ and $D$ the underlying division algebra. The index of $A$ equals $3^{i}$ for some $i=0, \ldots, 3$.

Assume $J_{3}(G)=(2,0)$. Then the Borel variety and $\mathrm{SB}(A)$ have a common upper motive. In particular, the Poincaré polynomial of this motive equals $\frac{t^{3^{j_{1}}}-1}{t-1}$. Hence, ind $A=3^{j_{1}}=9$.

Let $K=k\left(\mathrm{SB}_{3}(D)\right)$. Then by the index reduction formula ind $D_{K}=3$ (see [ScvB]). Therefore $J_{3}\left(G_{K}\right)=(1,0)$. (The second entry is zero because each entry in the $J$-invariant is non-increasing under field extensions.)

Since $J_{3}(G)=(2,0)$, the variety $X_{2}$ has no zero-cycles of degree coprime to 3 (see Lemma 8.2). Therefore by Lemma $8.8\left(X_{2}\right)_{K}$ has a zero-cycle of degree $1 \mathrm{mod}$ 3.

On the other hand, since by the index reduction formula ind $D_{k\left(X_{2}\right)}=3$ (see [MPW]), the variety $\mathrm{SB}_{3}(D)_{k\left(X_{2}\right)}$ has a rational point. Thus, by Lemma 4.9 the motives $\mathcal{U}\left(X_{2}\right)$ and $\mathcal{U}\left(\mathrm{SB}_{3}(D)\right)$ are isomorphic.

By Lemma $8.3 \operatorname{dim} \mathcal{U}\left(X_{2}\right)=20$. On the other hand,

$$
\operatorname{dim} \mathcal{U}\left(\mathrm{SB}_{3}(D)\right) \leq \operatorname{dim} \mathrm{SB}_{3}(D)=\operatorname{dim} \operatorname{Gr}(3,9)=18<20,
$$

which is a contradiction.

\section{Reduction to CHARACTERISTIC Zero}

We now prove a general mechanism for transferring results from characteristic 0 to a field of prime characteristic.

Fix a prime number $\ell$ and $m \geq 1$. Construct a complete discrete valuation ring $R$ with residue field $k$ of characteristic $p$ (possibly equal to 0 or $\ell$ ) and fraction field $K$ of characteristic zero. In case $\ell=p$, we enlarge $R$ if necessary to include the $\ell^{m}$-th roots of unity. We have a split exact sequence:

$$
0 \rightarrow H^{d+1}\left(k, \mathbb{Z} / \ell^{m} \mathbb{Z}(d)\right) \stackrel{i_{k}^{K}}{\longrightarrow} H_{\mathrm{nr}}^{d+1}\left(K, \mu_{\ell^{m}}^{\otimes d}\right) \stackrel{\partial_{K}}{\longrightarrow} H^{d}\left(k, \mathbb{Z} / \ell^{m} \mathbb{Z}(d-1)\right) \rightarrow 0
$$

where $H_{\mathrm{nr}}^{d+1}$ denotes the subgroup of elements $x$ such that $n x$ is killed by the maximal unramified extension of $K$ for some $n$ not divisible by $p$, see [GaMS, p. 18] if $p \neq \ell$ and [Kato 82, Th. 3 and p. 235] if $p=\ell$. The explicit formulas for $i_{k}^{K}$ shows that it sends symbols in $H^{d+1}\left(k, \mathbb{Z} / \ell^{m} \mathbb{Z}(d)\right)$ to symbols in ker $\partial_{K}$. When $m=1$, $[\mathrm{GaPe}, 16.1]$ gives the converse that symbols in ker $\partial_{K}$ are images of symbols in $H^{d+1}(k, \mathbb{Z} / \ell \mathbb{Z}(d))$.

9.1. Lemma. In the above notation an element $\xi \in H^{d+1}(k, \mathbb{Z} / \ell \mathbb{Z}(d))$ is a symbol over some finite extension of $k$ of degree not divisible by $\ell$ if and only if there is a finite extension of $K$ not divisible by $\ell$ over which $i_{k}^{K}(\xi)$ is a symbol.

Proof. The "if" direction is clear, using that symbols in the image of $i_{k}^{K}$ are images of symbols. For "only if", one immediately reduces to the case where the given extension $E$ of $k$ is purely inseparable. But, since $[E: k]$ is not divisible by $\ell$, we have $\ell \neq p$, and the mod- $\ell$ Galois cohomology groups over $k$ and $E$ are the same, so in that case $\xi$ is already a symbol over $k$. 
9.2. Lemma. If $\xi \in H^{d+1}(k, \mathbb{Z} / 2 \mathbb{Z}(d))$ is such that $\operatorname{res}_{L / k}(\xi)$ is a symbol for some odd-degree extension $L$ of $k$, then $\xi$ is a symbol.

Proof. If char $k \neq 2$, the claim concerns the Galois cohomology group $H^{d+1}(k, \mathbb{Z} / 2 \mathbb{Z})$, and the lemma is a result of Rost [Ro99]. Otherwise, char $k=2$ and we take $R$ and $K$ as above with $\ell=2$ and $m=1$. Combining Rost's result and the previous lemma completes the proof.

Here is the promised reduction:

9.3. Proposition. Let $G$ be a simple simply connected linear algebraic group over $k$ and let $\ell^{m}$ be the largest power of the prime $\ell$ dividing the order of the Rost invariant $r_{G}$. Define $R$ and $K$ as above. Then:

(1) There is a simple simply connected linear algebraic group $H$ over $K$ that has the same Dynkin type and the same Tits index as $G$.

(2) For every $\xi \in H^{1}(k, G)$, there is a $\zeta \in H^{1}(K, H)$ so that:

(a) The mod- $\ell$ component of $r_{G}(\xi)$ is zero in $H^{3}\left(k, \mathbb{Z} / \ell^{m} \mathbb{Z}(2)\right.$ ) (resp., a symbol in $H^{3}(k, \mathbb{Z} / \ell \mathbb{Z}(2))$ if and only if the mod- $\ell$ component of $r_{H}(\zeta)$ is zero in $H^{3}\left(K, \mathbb{Z} / \ell^{m} \mathbb{Z}(2)\right.$ ) (resp., a symbol in $H^{3}(K, \mathbb{Z} / \ell \mathbb{Z}(2))$ ). If the mod- $\ell$ component of $r_{G}(\xi)$ is a sum of $\leq r$ symbols in $H^{3}\left(k, \mathbb{Z} / \ell^{m} \mathbb{Z}(2)\right)$ with a common slot, then the mod- $\ell$ component of $r_{H}(\zeta)$ is a sum of $\leq r$ symbols in $H^{3}\left(K, \mathbb{Z} / \ell^{m} \mathbb{Z}(2)\right)$ with a common slot.

(b) For every finite extension $L / K$, the Tits indexes of the twisted forms $\left({ }_{\zeta} H\right)_{L}$ and $\left.{ }_{\xi} G\right)_{\bar{L}}$ are equal, where $\bar{L}$ is the residue field of $L$.

(c) For $X_{\Theta}^{\zeta}$ a twisted flag variety for ${ }_{\zeta} H$ and $X_{\Theta}^{\xi}$ the corresponding variety for ${ }_{\xi} G$,

$$
\operatorname{deg} \mathrm{CH}_{0} X_{\Theta}^{\zeta}=\operatorname{deg} \mathrm{CH}_{0} X_{\Theta}^{\xi}
$$

as subgroups of $\mathbb{Z}$.

Proof. We can find a semisimple group scheme $\mathcal{G}$ over $R$ of the same Dynkin type as $G$ whose special fiber is $G$ and whose generic fiber $\mathcal{G}_{K}$ is also of the same Dynkin type as $G$. Denote it by $H$. One can lift $\xi$ to a class in $H_{\text {ét }}^{1}(R, \mathcal{G})$ which we also denote by $\xi$. Let $\zeta$ be the image of $\xi$ in $H^{1}\left(K, \mathcal{G}_{K}\right)$. By [Gi00, Theorem 2] one has a commutative diagram

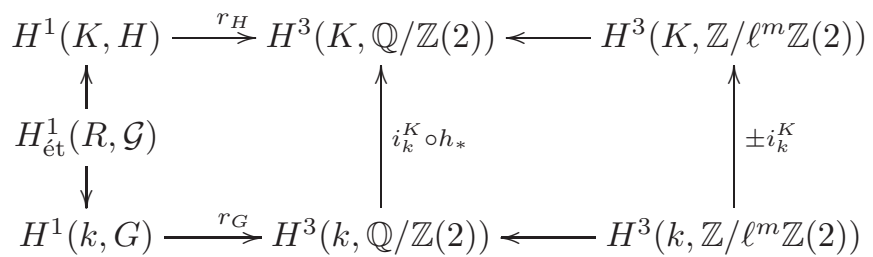

where $h_{*}$ is an automorphism that restricts to \pm 1 on $H^{3}\left(k, \mathbb{Z} / \ell^{m} \mathbb{Z}\right)$, hence the first sentence of (a). For the second sentence, the explicit formulas for $i_{k}^{K}$ show that it sends a sum of $\leq r$ symbols with a common slot to a sum of $\leq r$ symbols with a common slot.

The Tits indexes of $\left.{ }_{\zeta} H\right)_{L}$ and $\left({ }_{\xi} G\right)_{\bar{L}}$ are the same by [DG, Exposé 26, 7.15], hence (b). It follows that $\operatorname{deg} \mathrm{CH}_{0} X_{\Theta}^{\zeta} \subseteq \operatorname{deg} \mathrm{CH}_{0} X_{\Theta}^{\xi}$. For equality, in view of (b) it suffices to check that for every finite extension $k^{\prime}$ of $k$, there exists an extension $K^{\prime}$ of $K$ with residue field $k^{\prime}$ such that $\left[K^{\prime}: K\right]=\left[k^{\prime}: k\right]$, which is a routine exercise because the valuation is Henselian. 
Claim (2b) in the proposition is well known in some special cases, for example when $G$ is the special orthogonal group of a quadratic form as in [Lam, Prop. VI.1.9(1)] or $G$ is $\mathrm{PGL}_{n}$ as in [JaW, Th. 2.8(b)] (which does not require the valuation to be discrete).

We illustrate the proposition by applying it in its typical manner. We number the simple roots of $\mathrm{E}_{7}$ as in (10.1).

9.4. Corollary. Let $G$ be a simple algebraic group of type $\mathrm{E}_{7}$ over a field $k$. If the twisted flag variety $X_{7}$ has a zero-cycle of odd degree, then $X_{7}$ has a $k$-point.

Proof. The claim holds when char $k=0$ by [GiSe, Cor. 3.5], so assume char $k$ is prime. For $K$ as defined earlier in this section, Prop. 9.3(2c) gives that $X_{7}(K)$ has a zero-cycle of odd degree, hence $X_{7}(K) \neq \emptyset$ by ibid., hence $X_{7}(k) \neq \emptyset$ by Prop. 9.3(2b).

\section{Applications to the Rost invariant}

10a. Type $\mathrm{E}_{6}$. We now return to the setting of $\S 8$.

10.1. Lemma. Let $G$ be a group of inner type $\mathrm{E}_{6}$ over a field $k$. If $J_{3}(G)=(0,0)$, then $G$ is isotropic.

Proof. By [PSZ, Corollary 6.7] since $J_{3}(G)=(0,0), G$ splits over a field extension of $k$ of degree coprime to 3. Therefore the Tits algebra of $G$ (of degree 27) is split, so we may speak of the Rost invariant of $G$. Clearly, its 3-component must be zero.

If char $k \neq 2,3$, then by [Ro91] the variety $X_{1}$ has a rational point. Proposition 9.3 implies that the same holds over any field of prime characteristic. In particular, $G$ is isotropic.

10.2. Lemma. Let $G$ be a group of inner type $\mathrm{E}_{6}$ and $A$ a Tits algebra of $G$. Assume that ind $A \leq 3$. Then $G \times k(\mathrm{SB}(A))$ is isotropic if and only if $X_{2}$ has a zero-cycle of degree not divisible by 3.

Proof. Suppose first that char $k=0, G$ is anisotropic, $G_{k(\operatorname{SB}(A))}$ is isotropic, and every zero-cycle of $X_{2}$ has degree divisible by 3 . We know by Lemma 8.8, Corollary 8.10, and Lemma 10.1 that $j_{2}=1$. Since $G_{k(\operatorname{SB}(A))}$ is isotropic and $A_{k(\mathrm{SB}(A))}$ is split, $X_{2}$ has a zero-cycle over $k(\mathrm{SB}(A))$ of degree 1 or 2 , hence $J_{3}\left(G_{k(\operatorname{SB}(A))}\right)=(0,0)$ by Lemma 8.2 .

On the other hand, ind $A_{k\left(X_{\Delta}\right)}=1$. Therefore, the upper motives $\mathcal{U}\left(X_{\Delta}\right)$ and $\mathcal{U}(\mathrm{SB}(A))$ are isomorphic. Their Poincaré polynomials equal

$$
\left(1+t^{4}+t^{8}\right)\left(t^{3^{j_{1}}}-1\right) /(t-1)
$$

and $\left(t^{\text {ind } A}-1\right) /(t-1)$ respectively. In particular, they are not equal for any values of $j_{1}$ and ind $A$. Contradiction, so the "only if" direction is proved if char $k=0$ and $G$ is anisotropic; this is the crux case.

If $G$ is isotropic, then it is split or has semisimple anisotropic kernel of type $2 \mathrm{~A}_{2}$ or $\mathrm{D}_{4}$. In the first two cases, $X_{2}$ has a rational point and in the third case it has a point over a quadratic extension of $k$. Thus we have proved "only if" when char $k=0$ or $G$ is isotropic.

So consider the case where char $k$ is a prime $p, G_{k(\mathrm{SB}(A))}$ is isotropic, and $G$ is anisotropic; in particular, $A$ is not split, hence, by our assumptions has index 3 . Then there is a simply connected isotropic group $G^{\prime}$ (with anisotropic kernel of 
type $\left.2 \mathrm{~A}_{2}\right)$ and a class $\eta \in H^{1}\left(k, G^{\prime}\right)$ such that $G$ is isomorphic to $G^{\prime}$ twisted by $\eta$. We control the mod-3 portion $r_{G^{\prime}}(\eta)_{3}$ of the Rost invariant of $\eta$, which belongs to $H^{3}(k, \mathbb{Z} / 3 \mathbb{Z}(2))$. Clearly, $G^{\prime}$ is split by $k(\mathrm{SB}(A))$, so our hypothesis on $G$ gives that $k(\mathrm{SB}(A))$ kills $r_{G^{\prime}}(\eta)_{3}$. It follows that $r_{G^{\prime}}(\eta)_{3}=(\zeta) \cdot[A]$ for some $\zeta \in k^{\times} / k^{\times 3}$ by [Pey] and [Ka98, Prop. 5.1], hence by [GaQu] we may replace $\eta$ by a twist by the class of a cocycle with values in the center of $G^{\prime}$ and so assume that $r_{G^{\prime}}(\eta)_{3}$ is zero.

One can find a simply-connected group $H$ of inner type $\mathrm{E}_{6}$ over a field $K$ of characteristic 0 lifting $G^{\prime}$ and $\zeta \in H^{1}(K, H)$ lifting $\eta$ as in Proposition 9.3. In particular, $r_{H}(\zeta)_{3}=0$. Denote by $A_{H}$ the Tits algebra of $H$. By [Ro91] the twisted form is isotropic over $K\left(\mathrm{SB}\left(A_{H}\right)\right)$, and, thus, by Proposition 9.3(2c) and the characteristic zero case, we have proved the "only if" part.

Now suppose that there is an extension $L / k$ of degree not divisible by 3 so that $X_{2}(L)$ is not empty. If $A$ has index 1 , then $J_{3}(G)=(0,0)$ by Lemma 8.2, and so $G$ is $k$-isotropic by Lemma 10.1. If $A$ has index 3, then $L \otimes_{k} k(\mathrm{SB}(A))$ is a field of dimension not divisible by 3 over $k(\operatorname{SB}(A))$, hence the "if" statement follows by the index 1 case.

10.3. Remark. In case char $k \neq 2$, one can use the Rost invariant to define a class $r(G) \in H^{3}(k, \mathbb{Z} / 2 \mathbb{Z})$ depending only on $G$, see [GaGi, $\S 7$ ]. If $L / k$ is an extension such that $X_{2}(L)$ is nonempty, then certainly $r(G)$ is killed by $L$, hence $[L: k] r(G)=0$. It follows that $\operatorname{deg} \mathrm{CH}_{0}\left(X_{2}\right)$ is contained in $o(r(G)) \mathbb{Z}$, for $o(r(G))$ the order of $r(G)$, which is 1 or 2 . One can show that the conditions in Proposition 10.2 are equivalent to $\operatorname{deg} \mathrm{CH}_{0}\left(X_{2}\right)=o(r(G)) \mathbb{Z}$.

10.4. Corollary. Lemma 8.8, Corollary 8.9, and Corollary 8.10 hold in any characteristic.

Proof. Clearly, it suffices to prove only Lemma 8.8, so assume $J_{3}(G)=(1,0)$. Then $G$ is split by an extension of degree not divisible by 9 [PSZ, Prop. 6.6], so ind $A=3$ and $J_{3}\left(G_{k(\mathrm{SB}(A))}\right)=(0,0)$. Therefore by Lemma $10.1 G_{k(\mathrm{SB}(A))}$ is isotropic and by Lemma $10.2 X_{2}$ has a zero-cycle of degree coprime to 3 .

10.5. Corollary. Let $G$ be a group of inner type $\mathrm{E}_{6}$ with Tits algebra A. If $G \times$ $k(\mathrm{SB}(A))$ is isotropic, then $A$ has index dividing 3.

Proof. Since Lemma 8.8 and Corollary 8.10 hold in any characteristic, we can repeat the first two paragraphs of the proof of Lemma 10.2 without any restriction on the characteristic of $k$ to see that $X_{2}$ has a zero-cycle of degree not divisible by 3 .

We summarize the relationship between the mod-3 $J$-invariant of $G$ and its Tits index and Tits algebra in Table 10A. We use here that by [Ju, Prop. 5.3] $j_{1}=1$ iff ind $A=3$.

10.6. Proposition. Let $G$ be a simply connected group of inner type $\mathrm{E}_{6}$ over $k$ such that $X_{2}$ has a zero-cycle of degree 1 . Write $Z$ for the center of $G$.

(1) The Rost invariant $r_{G}$ is injective on the image of $H^{1}(k, Z) \rightarrow H^{1}(k, G)$.

(2) For $\xi \in H^{1}(k, G)$, if the mod-3 component of the Rost invariant $r_{G}(\xi)$ is a symbol, then

$$
\operatorname{gcd}\{[L: k] \mid L \text { kills } \xi\}=o\left(r_{G}(\xi)\right)
$$




\begin{tabular}{|c|c|c|c|c|c|}
\hline$J_{3}(G)$ & $(0,0)$ & $(1,0)$ & $(0,1)$ & $(1,1)$ & $(2,1)$ \\
\hline Tits index of $G$ & split & & \multicolumn{3}{|c|}{$\cdots$ anisotropic $\cdots$} \\
\hline index of $A$ & 1 & 3 & 1 & 3 & 9 or 27 \\
\hline
\end{tabular}

TABLE 10A. Dictionary relating the mod-3 $J$-invariant of $G$, the Tits index of $G$ over a 3 -closure of $k$, and the Tits algebra $A$ of $G$

Proof. Write $A$ for the Tits algebra of $G$. If $A$ is split, then $G$ is split and $H^{1}(k, Z)$ has zero image in $H^{1}(k, G)$, so (1) holds. If $A$ has index 3 , then $H^{1}(k, Z)$ is identified with $k^{\times} / k^{\times 3}$ and the composition

$$
H^{1}(k, Z) \rightarrow H^{1}(k, G) \rightarrow H^{3}(k, \mathbb{Q} / \mathbb{Z}(2))
$$

is $x \mapsto \pm x \cdot[A]$ by [GaQu]. By twisting, it suffices to show that this map has zero kernel. But if $x \cdot[A]$ is zero, then $x$ is a reduced norm from $A$, i.e., there is a cubic extension $L$ of $k$ in $A$ so that $x=N_{L / k}(y)$ for some $y \in L$ by MerkurjevSuslin if $\operatorname{char} k \neq 3$ and by [Gi00, Th. 6a] if $\operatorname{char} k=3$. Now $L$ splits $A$, so $G$ is $L$-split and $y$ is in the kernel of $H^{1}(L, Z) \rightarrow H^{1}(L, G)$. As $G / Z$ is rational as a variety over $L$, the Gille-Merkurjev Norm Principle implies that $x$ is in the kernel of $H^{1}(k, Z) \rightarrow H^{1}(k, G)$, completing the proof of (1).

As for (2), one quickly reduces to the case where $r_{G}(\xi)$ is zero (because the mod2 and mod-3 components of $r_{G}(\xi)$ are symbols - for 2 this is by Lemma 9.2), $X_{2}$ has a rational point, and $A$ has index 3 . There is a cubic extension of $k$ splitting $A$, hence splitting $G$, hence killing $\xi$. On the other hand, ${ }_{\xi} G \times k(\mathrm{SB}(A))$ is split, so by Lemma 10.2 the ${ }_{\xi} G$-variety $X_{2}$ has a point over extensions $L_{1}, \ldots, L_{r}$ such that $\operatorname{gcd}\left\{\left[L_{i}: k\right]\right\}$ is not divisible by 3 . Over each $L_{i}, \xi$ is in the kernel of the map $H^{1}\left(L_{i}, G\right) \rightarrow H^{1}\left(L_{i}, G / Z\right)$ by Tits's Witt-type Theorem, so is equivalent to the class of a cocycle $z$ with values in $Z$. By (1), $\xi$ is killed by $L_{i}$. This proves (2).

10b. Type $\mathrm{E}_{7}$. For use in this subsection and the next, we recall that the simple roots of $\mathrm{E}_{7}$ and $\mathrm{E}_{8}$ are numbered like this:

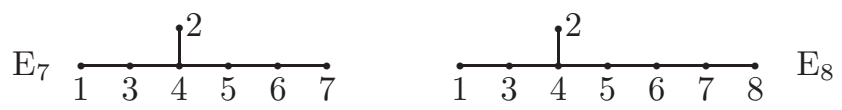

A group $G$ of type $\mathrm{E}_{7}$ has (essentially) one Tits algebra, as explained in [Ti71, 6.5.1]. It is a central simple algebra of exponent dividing 2 and index dividing 8 .

10.7. Proposition. Let $G$ be an anisotropic group of type $\mathrm{E}_{7}$ with Tits algebra $H$. If $G_{k(\mathrm{SB}(H))}$ is split, then ind $H=2$.

Proof. Let $J_{2}(G)=\left(j_{1}, j_{2}, j_{3}, j_{4}\right), j_{i}=0,1$, be the $J$-invariant of adjoint $\mathrm{E}_{7}$ (see [PSZ, Section 4.13]). Since $G$ is anisotropic and $G_{k(\mathrm{SB}(H))}$ is split, $j_{1}=1$ by [PS10, Proposition 4.2].

Moreover, the upper motives of the variety of Borel subgroups $X_{\Delta}$ and of $\mathrm{SB}(H)$ are isomorphic. Their Poincaré polynomials equal

$$
(1+t)\left(1+t^{3}\right)^{j_{2}}\left(1+t^{5}\right)^{j_{3}}\left(1+t^{9}\right)^{j_{4}} \quad \text { and } \quad \frac{t^{\text {ind } H}-1}{t-1} .
$$

Since they are equal, we have $j_{2}=j_{3}=j_{4}=0$ and ind $H=2$. 
The following lemma provides a crucial computation for the proof of Theorems 10.10 and 10.18 below, which settle Rost's question described in the introduction. The proof involves a computer calculation, which we did via two independent methods: the one described in section 5 and the one in [DuZ]. Alternatively, the paper [KonoI] computes the Steenrod operations on $\mathrm{Ch}\left(\bar{X}_{\Delta}\right)$, and presumably the computer calculation here could be replaced by an argument connecting their computation with $X_{7}$.

10.8. Lemma. Assume that the variety $X_{7}$ does not have a zero-cycle of odd degree, the Tits algebras of $G$ are split, and char $k \neq 2$. Then $\mathbb{F}_{2}(9)$ is a direct summand of the motive $\mathcal{U}\left(X_{7}\right)$ over $k\left(X_{7}\right)$.

Proof. Let $h \in \mathrm{Ch}^{1}\left(\bar{X}_{7}\right), e_{5} \in \mathrm{Ch}^{5}\left(\bar{X}_{7}\right)$, and $e_{9} \in \mathrm{Ch}^{9}\left(\bar{X}_{7}\right)$ denote some Schubert cycles. Then independently of the choice of these cycles, the elements $h^{9}, e_{5} h^{4}$, and $e_{9}$ form an $\mathbb{F}_{2}$-basis of $\mathrm{Ch}^{9}\left(\bar{X}_{7}\right)$. Note also that the cycle $h$ is rational, since the Tits algebras of $G$ are split.

We claim that the cycles $e_{5} h^{4}, e_{9}$, and $e_{5} h^{4}+e_{9}$ are not rational.

Indeed, a direct computation of Steenrod operations modulo 2 shows that

$$
e_{5} h^{5} \cdot S^{8}\left(e_{5} h^{4}\right)=e_{9} h \cdot S^{8}\left(e_{9}\right)=\left(e_{5} h^{5}+e_{9} h\right) S^{8}\left(e_{5} h^{4}+e_{9}\right)=\mathrm{pt},
$$

where pt denotes the class of a rational point on $\bar{X}_{7}$. Since by our assumptions $X_{7}$ has no zero-cycles of odd degree, the only rational cycle in $\mathrm{Ch}^{9}\left(\bar{X}_{7}\right)$ is $h^{9}$.

But the cycle $h^{9}$ does not lie in the first shell. Indeed, an explicit computation of the decomposition of [CGM, Theorem 7.5] for $\mathscr{M}\left(X_{7}\right)$ shows that over $k\left(X_{7}\right)$ its motive contains exactly the following Tate motives: $\mathbb{F}_{2}, \mathbb{F}_{2}(1), \mathbb{F}_{2}(9), \mathbb{F}_{2}(10)$, $\mathbb{F}_{2}(17), \mathbb{F}_{2}(18), \mathbb{F}_{2}(26)$, and $\mathbb{F}_{2}(27)$, and that the cycle from the dual codimension that corresponds to the Tate motive $\mathbb{F}_{2}(9)$ equals $Z_{[1,3,4,2,5,4,3,1,7,6,5,4,2,3,4,5,6,7]}$ in the notation of Section 5. A direct computation using Poincaré duality shows that this cycle is not dual to $h^{9}$.

Since generic points of direct summands of $X_{7}$ are rational, no shift of $\mathcal{U}\left(X_{7}\right)$ of $X_{7}$ starts in codimension 9 . Therefore the Tate motive $\mathbb{F}_{2}(9)$, which belongs to the first shell, must be a summand of $\mathcal{U}\left(X_{7}\right)_{k\left(X_{7}\right)}$.

10.9. Lemma. Assume that $G$ is anisotropic, the variety $X_{7}$ has no zero-cycles of odd degree, the Tits algebras of $G$ are split, and char $k \neq 2$. Then the height of $X_{1}$ equals 3 and $G_{k\left(X_{1}\right)}$ has semisimple anisotropic kernel of type $\mathrm{D}_{6}$.

Proof. The $(2,\{1\})$-indexes of $G$ are $\{1\}, \Psi:=\{1,6,7\}$, and $\Delta$, so by Example 4.7 the nonempty shells on $X_{1}$ are the first shell, which is contained in $\mathrm{SH}_{\leqslant \Psi}$, which is contained in the last shell.

By [PS10, Th. 5.7(6)] the varieties $X_{1}$ and $X_{7}$ are not generically split. Therefore by the Tits classification [Ti66] the height of $X_{1}$ is 2 or 3 . Assume that it is two. Then the upper motives $\mathcal{U}\left(X_{7}\right)$ and $\mathcal{U}\left(X_{1}\right)$ are isomorphic.

By Lemma 10.8 the motive $\mathcal{U}\left(X_{7}\right)$ has the property that $\mathbb{F}_{2}(9)$ is its direct summand over $k\left(X_{7}\right)$. On the other hand, a direct computation using [CGM, Th. 7.5] shows that $\mathbb{F}_{2}(9)$ is not a direct summand of the motive of $X_{1}$ over $k\left(X_{7}\right)$. Contradiction.

10.10. Theorem. Let $G_{0}$ be a split, simply connected group of type $\mathrm{E}_{7}, \xi \in H^{1}\left(k, G_{0}\right)$, and $G={ }_{\xi}\left(G_{0}\right)$. The following conditions are equivalent

(1) $6 r_{G_{0}}(\xi)=0$ and the mod-2 component of $r_{G_{0}}(\xi)$ is a symbol; 
(2) the $G$-variety $X_{7}$ has a rational point;

(3) the element $\xi$ lifts to $H^{1}\left(k, \mathrm{E}_{6}\right)$, where $\mathrm{E}_{6}$ stands for the split simply connected group of type $\mathrm{E}_{6}$.

This result settles the question raised in the last sentence of [Sp06], which asked whether there exists a criterion on $r_{G_{0}}(\xi)$ for whether (3) holds.

The proof uses the following notion: suppose $\alpha: H \rightarrow G_{0}$ is a homomorphism of absolutely simple simply connected groups. Then the composition $H^{1}(-, H) \stackrel{\alpha}{\rightarrow}$ $H^{1}\left(-, G_{0}\right) \stackrel{r_{G_{0}}}{\longrightarrow} H^{3}(-, \mathbb{Q} / \mathbb{Z}(2))$ equals $n_{\alpha} r_{H}$ for some nonnegative integer $n_{\alpha}$ called the Dynkin index of $\alpha$, see [GaMS, pp. 122, 123] for its basic properties. It can be calculated from root system data as follows. Let $T$ be a maximal torus of $G_{0}$ and $U$ be a maximal torus in $H$ such that $\alpha(U) \subseteq T$. There is a unique Weyl-invariant quadratic form $q$ on the coroot lattice $\operatorname{Hom}\left(\mathbb{G}_{m}, T\right)$ that takes the value 1 on short coroots, and the Dynkin index $n_{\alpha}$ is the value of $q$ on the image under $\alpha$ of a short coroot in $\operatorname{Hom}\left(\mathbb{G}_{m}, U\right)$.

Proof of Theorem 10.10. Assume (1), and that (2) fails; we seek a contradiction. By Proposition 9.3 we may assume that char $k=0$. A $X_{7}$ has no rational point, it has no zero-cycle of odd degree as in Cor. 9.4.

By Lemma 10.9, the anisotropic kernel of $G_{k\left(X_{1}\right)}$ has type $\mathrm{D}_{6}$ and, thus, equals $\operatorname{Spin}(q)$ for a 12-dimensional quadratic form $q$ with trivial discriminant and trivial Clifford invariant. Because the inclusion $\mathrm{D}_{6} \subset \mathrm{E}_{7}$ has Dynkin index 1, the Arason invariant of $q$ is also a symbol. This gives a contradiction with [Ga09a, Lemma 12.5], hence $(1) \Rightarrow(2)$.

(3) obviously implies (1). Assume (2). By Tits's Witt-type theorem, $\xi$ is equivalent to the class of a cocycle taking values in the parabolic subgroup $P_{7}$. Let $L$ be the Levi part of $P_{7}$. By [DG, Exp. XXVI, Cor. 2.3] $H^{1}\left(k, P_{7}\right)=H^{1}(k, L)$. Then $\xi \in H^{1}(k, L)$ comes from $H^{1}\left(k, \mathrm{E}_{6}\right)$ by the exact sequence

$$
1 \rightarrow \mathrm{E}_{6} \rightarrow L \rightarrow \mathbb{G}_{m} \rightarrow 1
$$

and by Hilbert 90 .

The split simply connected group $\mathrm{E}_{6}$ contains a split group $\mathrm{G}_{2}$ of that type, and the inclusion $\mathrm{G}_{2} \subset \mathrm{E}_{6}$ has Dynkin index 1 .

10.11. Corollary. The map $H^{1}\left(k, \mathrm{G}_{2}\right) \rightarrow H^{1}\left(k, G_{0}\right)$ identifies $H^{1}\left(k, \mathrm{G}_{2}\right)$ with the subset

$$
\left\{\xi \in H^{1}\left(k, G_{0}\right) \mid r_{G_{0}}(\xi) \text { is a symbol in } H^{3}(k, \mathbb{Z} / 2 \mathbb{Z}(2))\right\} \text {. }
$$

For each such $\xi$, the kernel of the Rost invariant $H^{1}\left(k, \xi\left(G_{0}\right)\right) \rightarrow H^{3}(k, \mathbb{Z} / 12 \mathbb{Z}(2))$ is zero.

In the statement, $G_{0}$ is split simply connected of type $\mathrm{E}_{7}$, as in Theorem 10.10. But note that the statement holds verbatim if $G_{0}$ is instead taken to be $\mathrm{E}_{6}$.

Proof. The Rost invariant $r_{\mathrm{G}_{2}}$ identifies $H^{1}\left(k, \mathrm{G}_{2}\right)$ with the set of symbols in $H^{3}(k, \mathbb{Z} / 2 \mathbb{Z}(2))$, cf. [GaMS, p. 44]. Hence, as the total inclusion $\mathrm{G}_{2} \subset \mathrm{E}_{6} \subset G_{0}$ has Dynkin index 1 , the image of $H^{1}\left(k, \mathrm{G}_{2}\right)$ is contained in the displayed set. Conversely, if $\xi, \xi^{\prime}$ are in the displayed set and $r_{G_{0}}(\xi)=r_{G_{0}}\left(\xi^{\prime}\right)$, then $\xi, \xi^{\prime}$ come from $H^{1}\left(k, \mathrm{E}_{6}\right)$ by Theorem 10.10 , hence $\xi=\xi^{\prime}$ by the analogous property for $\mathrm{E}_{6}$. The second claim follows from the first by twisting. 
10.12. Remark. This corollary includes as a special case that ker $r_{G_{0}}=0$. So it gives a fourth proof of this statement, with the first three being Garibaldi (2001), Chernousov (2003), and Petrov-Semenov [PS10].

It is conjectured that the Rost invariant $H^{1}\left(k,{ }_{\xi}\left(G_{0}\right)\right) \rightarrow H^{3}(k, \mathbb{Z} / 12 \mathbb{Z}(2))$ has zero kernel whenever $\xi$ satisfies Theorem 10.10(1), see [Ga09b, 11.11].

10.13. Corollary. If char $k=0, k(\sqrt{-1})$ has cohomological dimension $\leq 2$, and $r_{G_{0}}(\xi)$ is a symbol in $H^{3}(k, \mathbb{Z} / 2 \mathbb{Z})$, then the natural map

$$
H^{1}\left(k, \xi\left(G_{0}\right)\right) \rightarrow \prod_{\text {orderings } v \text { of } k} H^{1}\left(k_{v}, \xi\left(G_{0}\right)\right)
$$

has zero kernel.

That is, the "Hasse Principle Conjecture II" holds for the group $\xi\left(G_{0}\right)$. This is new. The analogous statement in prime characteristic is Serre's "Conjecture II", which is known for these groups by, e.g., [Gi01].

Proof of Cor. 10.13. The hypothesis on $k$ gives that

$$
H^{3}(k, \mathbb{Q} / \mathbb{Z}(2))=H^{3}(k, \mathbb{Z} / 2 \mathbb{Z}),
$$

and the claim is obvious from Corollary 10.11 and the injectivity of the map $H^{3}(k, \mathbb{Z} / 2 \mathbb{Z}) \rightarrow \prod H^{3}\left(k_{v}, \mathbb{Z} / 2 \mathbb{Z}\right)$.

We can also prove a new case of the local-global principle studied in $[\mathrm{PaPr}]$. A global field $k$ is a number field or a finite extension $\mathbb{F}_{p}(t)$. We write $k_{v}$ for the completion of $k$ with respect to a valuation $v$.

10.14. Corollary. Let $C$ be a proper, smooth, and geometrically integral curve over a global field $k$. If

(1) $G$ is the base change to $k(C)$ of a simply connected group of type $\mathrm{E}_{7}$ with trivial Tits algebras over $k$; or

(2) $G={ }_{\xi}\left(G_{0}\right)$ for some $\xi \in H^{1}\left(k(C), G_{0}\right)$ such that $r_{G_{0}}(\xi)$ is a symbol in $H^{3}(k(C), \mathbb{Z} / 2 \mathbb{Z}(2))$,

then the natural map

$$
H^{1}(k(C), G) \rightarrow \prod_{\text {valuation v of } k} H^{1}\left(k_{v}(C), G\right)
$$

has zero kernel.

Proof. Suppose we are in case (2) and $x \in H^{1}(k(C), G)$ has zero image in $H^{1}\left(k_{v}(C), G\right)$ for all $v$. Then $r_{G}(x)$ has zero image under

$$
H^{3}(k(C), \mathbb{Z} / 12 \mathbb{Z}(2)) \rightarrow \prod_{v} H^{3}\left(k_{v}(C), \mathbb{Z} / 12 \mathbb{Z}(2)\right),
$$

hence $r_{G}(x)$ equals zero by [Kato 86, Th. 0.8(2)], and Corollary 10.11 gives the claim.

In case (1), let $\xi \in H^{1}\left(k, G_{0}\right)$ be such that $G={ }_{\xi} G_{0} \times k(C)$. Then $r_{G_{0}}(\xi)$ belongs to $H^{3}(k, \mathbb{Q} / \mathbb{Z}(2))=H^{3}(k, \mathbb{Z} / 2 \mathbb{Z}(2))$, so it is necessarily a symbol, i.e., (1) is a special case of $(2)$. 
With this same kind of proof, combined with the arguments from [HHK, §4.3], one can also prove a local-global principle for $\xi\left(G_{0}\right)$ as in Corollaries 10.13 and 10.14, but with the field replaced by the function field of a curve over a complete discretely valued field.

10.15. Lemma. Let $q$ be a regular 12-dimensional quadratic form with trivial discriminant over a field $k$ with char $k \neq 2$ such that the respective special orthogonal group has J-invariant $(0,1,0)$. Then $q$ is isotropic.

Proof. Assume that $q$ is anisotropic.

Let $G$ be the orthogonal group corresponding to $q$. By [PS10, Prop. 4.2] the Clifford invariant of $q$ is trivial. Therefore by the classification of 12-dimensional quadratic forms $q$ has splitting pattern $(2,4)$. Let $Q=X_{1}$ be the projective quadric given by $q=0$ and $h \in \mathrm{Ch}^{1}\left(\bar{X}_{1}\right)$ the unique Schubert cycle.

By Example 4.6, there are exactly two (non-empty) shells on $Q$, namely, $\mathrm{SH}_{\leqslant\{1\}}$ (the first shell) and $\mathrm{SH}_{\leqslant\{3\}}$. The powers $h^{i} \in \mathrm{Ch}^{i}\left(\bar{X}_{1}\right)$ are rational and lie in the first shell if $i=0,1$ and in $\mathrm{SH}_{\leqslant\{3\}} \backslash \mathrm{SH}_{\leqslant\{1\}}$ if $i=2,3,4,5$.

Since $J_{2}(G)=(0,1,0)$, the Poincaré polynomial of the upper motive $\mathcal{U}\left(X_{\Delta}\right)$ of the Borel variety equals $t^{3}+1$. Moreover, since $q$ has height two, $\mathcal{U}\left(X_{\Delta}\right)_{k(Q)}$ is indecomposable.

We have the following motivic decomposition over $k(Q)$ :

$$
\mathscr{M}\left(Q_{k(Q)}\right) \simeq \oplus_{i=0,1,9,10} \mathbb{F}_{2}(i) \bigoplus \oplus_{i=2}^{5} \mathcal{U}\left(X_{\Delta}\right)_{k(Q)}(i) .
$$

So, all conditions of Proposition 7.7 are satisfied and the parameter $b$ of that Proposition equals 9. This is a contradiction, since $9 \neq 2^{n}-1$ for any $n$. (In the proof of Proposition 7.7 in case $X$ is a projective quadric, one can use [Vilo, Theorem 2.1] instead of Corollary 7.6. Then the restriction char $k=0$ is substituted by the restriction char $k \neq 2$.)

10.16. Proposition. Let $G$ be an adjoint group of type $\mathrm{E}_{7}$ with $J_{2}(G)=(0,1,0,0)$ over a field $k$ with char $k \neq 2$. Then $X_{7}$ has a rational point.

Proof. Since $j_{1}=0$, the Tits algebras of $G$ are split.

If $G$ is isotropic with anisotropic kernel of type $\mathrm{D}_{6}$, then we get a contradiction with Lemma 10.15, so assume that $G$ is anisotropic. Then $X_{7}$ has no zero-cycles of odd degree by Corollary 9.4, so by Lemma 10.9 the height of $X_{1}$ equals 3 and the semisimple anisotropic kernel $G^{\prime}$ of $G_{k\left(X_{1}\right)}$ has type $\mathrm{D}_{6}$.

Since the $J$-invariant is non-increasing under field extensions and since $G_{k\left(X_{1}\right)}$ is not split, the $J$-invariant of $G_{k\left(X_{1}\right)}$ also equals $(0,1,0,0)$. Therefore by [PSZ, Cor. 5.19] we have $J_{2}\left(G^{\prime}\right)=(0,1,0)$, and again we get a contradiction with Lemma 10.15 .

10.17. Corollary. Let $C$ be a smooth projective irreducible curve over $\mathbb{Q}_{p}, G_{0}$ be a split simply-connected group of type $\mathrm{E}_{7}$ over $\mathbb{Q}_{p}(C)$ and $\xi \in H^{1}\left(\mathbb{Q}_{p}(C), G_{0}\right)$. Then: $6 r_{G_{0}}(\xi)=0$ iff $\xi\left(G_{0}\right)$ is isotropic.

Proof. Note first that the order of the Rost invariant $r_{G_{0}}$ is 12 and that "if" is easy.

Assume $6 r_{G_{0}}(\xi)=0$. Then the mod-4 component of the Rost invariant of $\xi$ lies in $H^{3}(k(C), \mathbb{Z} / 2)$ and so is a symbol by [PaSu98, Th. 3.9] or [Leep]. Theorem 10.10 gives that $\xi\left(G_{0}\right)$ is isotropic. 
We remark that, using the theory of Bruhat-Tits buildings J. Tits shows in [Ti90, Proposition 2(B)] that there is an anisotropic group of type $\mathrm{E}_{7}$ with trivial Tits algebras over $\mathbb{Q}_{p}(t)$. That is, there exists $\xi \in H^{1}\left(\mathbb{Q}_{p}(t), G_{0}\right)$ such that ${ }_{\xi}\left(G_{0}\right)$ is anisotropic.

10.18. Theorem. Let $G_{0}$ be a split, simply connected group of type $\mathrm{E}_{7}$ over a field $k, \xi \in H^{1}\left(k, G_{0}\right)$, and $G={ }_{\xi}\left(G_{0}\right)$. The following conditions are equivalent

(1) there is an odd-degree extension $L / k$ so that $r_{G_{0}}\left(\xi_{L}\right)$ is a sum of two symbols in $H^{3}(L, \mathbb{Z} / 2 \mathbb{Z}(2))$ with a common slot;

(2) the $G$-variety $X_{1}$ has a zero-cycle of odd degree;

(3) $G$ becomes isotropic over an odd-degree extension of $k$.

Proof. The implication (2) $\Rightarrow(3)$ is trivial and (3) $\Rightarrow(1)$ is [Ga09b, p. 70, Prop. A.1]. So assume (1); we prove (2), We may replace $k$ with $L$ and so assume that $r_{G_{0}}(\xi)$ is a sum of two symbols in $H^{3}(k, \mathbb{Z} / 2 \mathbb{Z}(2))$. We may assume that char $k=0$ by Proposition 9.3.

The $J$-invariant $J_{2}(G)$ is $(0,0,0),(1,0,0),(1,1,0)$, or $(1,1,1)$ because $G$ is simply connected. In the first two cases, $X_{7}$ has a rational point (by Cor. 9.4 and Prop. 10.16 respectively), hence (2). So we can assume that $J_{2}(G)=\left(1,1, j_{3}\right)$ for some $j_{3}$.

By hypothesis, there is a regular quadratic form $q$ over $k$ of dimension 12 whose Arason invariant equals $r_{G_{0}}(\xi)$. We assume that $q$ is anisotropic, for otherwise $r_{G_{0}}(\xi)$ is a symbol and $X_{7}$ has a rational point by Theorem 10.10, hence (2). We denote the corresponding projective quadric by $Q$. Over $k\left(X_{7}\right)$ the Rost invariant $r_{G_{0}}(\xi)$ is a symbol, hence the form $q$ is isotropic over $k\left(X_{7}\right)$. Conversely, the Rost invariant of $\xi$ over $k(Q)$ is a symbol, so by Theorem $10.10 X_{7}$ has a $k(Q)$ point. Therefore the upper motives $\mathcal{U}\left(X_{7}\right)$ and $\mathcal{U}(Q)$ are isomorphic. Moreover, $\mathscr{M}(Q) \simeq \mathcal{U}(Q) \oplus \mathcal{U}(Q)(1)$. Therefore, since $X_{7}$ has height 2, we have

$$
\mathscr{M}\left(X_{7}\right) \simeq \mathcal{U}(Q) \oplus \mathcal{U}(Q)(1) \oplus \mathcal{U}(Q)(17) \oplus \mathcal{U}(Q)(18) \oplus \oplus_{i \in I} \mathcal{U}\left(X_{\Delta}\right)(i),
$$

where $I$ is some multiset of indexes. The Poincaré polynomial of $\mathcal{U}\left(X_{\Delta}\right)$ equals

$$
\left(t^{3}+1\right)\left(t^{5}+1\right)\left(t^{9}+1\right)^{j_{3}}
$$

and $P\left(X_{7}, t\right)-\left(1+t+t^{17}+t^{18}\right) P(\mathcal{U}(Q), t)$ is divisible by $P\left(\mathcal{U}\left(X_{\Delta}\right), t\right)$. An easy computation shows then that $j_{3}=0$.

Consider now the variety $X_{1}$ over $K:=k\left(X_{1}\right)$. A direct computation using [CGM, Theorem 7.5] gives the following decomposition over $K$ :

$$
\mathscr{M}\left(X_{1}\right) \simeq \mathbb{F}_{2} \oplus \mathscr{M}\left(X_{3}^{\prime}\right)(1) \oplus \mathscr{M}\left(X_{6}^{\prime}\right)(8) \oplus \mathscr{M}\left(X_{3}^{\prime}\right)(17) \oplus \mathbb{F}_{3}(33),
$$

where $X_{3}^{\prime}$ and $X_{6}^{\prime}$ are $\operatorname{Spin}(q)$-homogeneous varieties of types 3 and 6 (here the enumeration of simple roots comes from the embedding $\mathrm{D}_{6}<\mathrm{E}_{7}$, i.e., $X_{3}^{\prime}$ is a connected component of the maximal orthogonal Grassmannian and $X_{6}^{\prime}$ is the variety of isotropic planes).

The variety $X_{3}^{\prime}$ is generically split. Therefore $\mathscr{M}\left(X_{3}^{\prime}\right)$ is a direct sum over $k$ of Tate twists of the motive $\mathcal{U}\left(X_{\Delta}\right)$. The variety $X_{6}^{\prime}$ has height 2 and is a direct sum over $k$ of Tate shifts of the motives $\mathcal{U}\left(X_{\Delta}\right)$ and $\mathcal{U}(Q)$.

But $J_{2}\left(G_{k\left(X_{1}\right)}\right)=(1,1,0)$ by Lemma 10.9 and Proposition 10.16. Therefore the motives $\mathcal{U}\left(X_{\Delta}\right)_{k\left(X_{1}\right)}$ and $\mathcal{U}(Q)_{k\left(X_{1}\right)}$ are indecomposable. If $X_{1}$ has no zero-cycles of odd degree, then we can apply Proposition 7.7, which gives a contradiction, since $33 \neq 2^{n}-1$ for any $n$. Therefore, $(1) \Rightarrow(2)$. 
10.19. Remark. For $G={ }_{\xi}\left(G_{0}\right)$ of type $\mathrm{E}_{7}$, it is known that (1) for any extension $L$ of $k$ such that $X_{7}(L) \neq \emptyset$, we have $\operatorname{res}_{L / k}\left(4 r_{G_{0}}(\xi)\right)=0$ iff $X_{1} \times L$ has a zero-cycle of degree not divisible by 3 (by [Ro91] and Prop. 9.3) and (2) there exists a separable extension $K$ of $k$ of dimension 1 or 2 such that $X_{7}(K) \neq \emptyset$ (by [Ga09b, 12.13] and Prop. 9.3). As $4 r_{G_{0}}(\xi) \in H^{3}(k, \mathbb{Z} / 3 \mathbb{Z}(2))$, combining these observations gives: $X_{1}$ has a zero-cycle of degree 1 iff $X_{1}$ has a zero-cycle of odd degree and $4 r_{G_{0}}(\xi)=0$.

Table 10B summarizes what we have proved about the relationships between the Rost invariant, and the Tits index for groups of type $\mathrm{E}_{7}$ at the prime 2; it also gives a description of $J$-invariant for simply connected groups of type $\mathrm{E}_{7}$ for fields of characteristic 0 . The equivalence for the $J$-invariant $(1,1,0)$ follows from the proof of Theorem 10.18 .

\begin{tabular}{|c|c|c|c|c|}
\hline$J_{2}(G)$ & $(0,0,0)$ & $(1,0,0)$ & $(1,1,0)$ & $(1,1,1)$ \\
\hline Tits index of $G$ & split & $\odot \cdot 1.0 \odot$ & $\odot \cdot 1$ & anisotropic \\
\hline$r_{G_{0}}(\xi)$ & 0 & $\begin{array}{l}\text { nonzero symbol in } \\
H^{3}(K, \mathbb{Z} / 2 \mathbb{Z}(2))\end{array}$ & $\begin{array}{l}\text { sum of two symbols } \\
\text { with a common slot in } \\
H^{3}(K, \mathbb{Z} / 2 \mathbb{Z}(2))\end{array}$ & otherwise \\
\hline
\end{tabular}

TABLE 10B. Dictionary relating the mod-2 $J$-invariant of $G$, the Tits index of $G$ over a 2-closure $K$ of $k$, and the Rost invariant $r_{G_{0}}\left(\xi_{K}\right)$, for $G_{0}$ split simply connected of type $\mathrm{E}_{7}$.

10.20. Remark. For completeness' sake, we mention the analogous results for a group $G$ of type $\mathrm{E}_{7}$ at the prime 3 . (The case of primes $>3$ being trivial.) There is an extension $L$ of $k$ of degree not divisible by 3 over which $G$ has trivial Tits algebras and the homogeneous variety $X_{7}$ has a rational point [Ga09b, 13.1]. It follows that the mod-3 component of $r\left(G_{L}\right)$ is a symbol. The mod-3 component of $r\left(G_{L}\right)$ is zero iff $X_{1,6,7}$ has an $L$-point.

10c. Type $\mathrm{E}_{8}$. Recall the following known result:

10.21. Proposition. Let $G_{0}$ be a split group of type $\mathrm{E}_{8}$ over a field $k, \xi \in H^{1}\left(k, G_{0}\right)$, $G={ }_{\xi}\left(G_{0}\right)$ and $p$ an odd prime. If the mod-p component of $r_{G_{0}}(\xi)$ is zero, then $G$ is split over a field extension of degree not divisible by $p$.

The proposition is trivial for $p \geq 11$ and $p=7$ amounts to noting that 7 does not divide 120, see [Ti92, p. 1135]. The cases $p=3,5$ are more substantial and are the main results of two papers of Chernousov, see [C95] or [Ga09b, Prop. 15.5] for the mod- 5 case and [C10] for the mod- 3 case. We give a short proof of the $p=3$ case using the methods of this paper.

Proof of Prop. 10.21 for $p=3$. By Proposition 9.3 we can assume that char $k=0$. Replacing $k$ by an extension of degree coprime to 3, we can assume that the Rost invariant $r_{G_{0}}(\xi)$ is zero.

Consider the variety $X$ of parabolic subgroups of $G$ of type 7 . By the classification of Tits indexes, $G$ has a parabolic of type 8 over $k(X)$, hence the semisimple anisotropic kernel of $G_{k(X)}$ is contained in a simply connected subgroup of type 
$\mathrm{E}_{6}$. But the Rost invariant of the split $\mathrm{E}_{6}$ has zero kernel, so it follows that $X$ is generically split.

Therefore by [PS10, Th. 5.7] $J_{3}(G)=(0,0)$, hence by [PS10, Prop. 3.9(3)] $G$ splits over a field extension of degree coprime to 3 .

The conclusion of Prop. 10.21 is false in general for the omitted prime $p=2$, e.g., in case $k=\mathbb{R}$. For $p=2$, one needs to inspect also the degree 5 invariant constructed in [Sem].

10.22. Lemma. Let $G$ be a group of type $\mathrm{E}_{8}$ over a field $k$ with char $k=0$. If $J_{3}(G)=(1,0)$, then $X_{8}$ is isotropic over a field extension of degree coprime to 3 .

Proof. Assume that $X_{8}$ has no zero-cycles of degree coprime to 3. By [PS10, Theorem 5.7(8)] $X_{8}$ is not generically split. Therefore, since $J_{3}(G)=(1,0)$, the motive $\mathcal{U}\left(X_{\Delta}\right)_{k\left(X_{8}\right)}$ is indecomposable.

We have the following motivic decomposition over $k\left(X_{8}\right)$ :

$$
\mathscr{M}\left(X_{8}\right) \simeq \oplus_{i=0,1,28,29,56,57} \mathbb{F}_{3}(i) \oplus\left(\oplus_{j \in J} \mathcal{U}\left(X_{\Delta}\right)(j)\right)
$$

for some multiset of indexes $J$.

Moreover, the Picard group of $X_{8}$ is rational, since the Tits algebras of $G$ are split. It follows that the (unique) generator of the Picard group lies in the first shell. This leads to a contradiction with Proposition 7.7 , since $28 \neq\left(3^{n}-1\right) / 2$ for any $n$.

With Lemma 10.22 in hand, we can significantly strengthen Prop. 10.21 by giving criteria for $r_{G_{0}}(\xi)$ to be a symbol over an extension of degree not divisible by some odd prime $p$. For $p \geq 5$, this happens for every $\xi$ (see [Ga09b, 14.7, 14.13] for the case $p=5)$. For $p=3$, we have:

10.23. Theorem. Let $G_{0}$ be a split group of type $\mathrm{E}_{8}$ over a field $k, \xi \in H^{1}\left(k, G_{0}\right)$, and $G={ }_{\xi}\left(G_{0}\right)$. The following conditions are equivalent:

(1) $r_{G_{0}}(\xi)$ is a symbol over a field extension of degree coprime to 3 ;

(2) The $G$-homogeneous variety $X_{7,8}$ is isotropic over a field extension of degree coprime to 3;

(3) $G$ is isotropic over a field extension of degree coprime to 3.

Proof. We assume (1) and prove (2). Without loss of generality we can assume that the even and the mod-5 components of the Rost invariant of $\xi$ are zero.

By Proposition 9.3 we can assume that $\operatorname{char} k=0$. Assume $r_{G_{0}}(\xi)$ is a symbol over a field extension of degree coprime to 3; by Prop. 10.21 we can assume that it is not zero. Consider its generic splitting variety $D$. The upper motive of $D$ is a generalized Rost motive $R$ with Poincaré polynomial $1+t^{4}+t^{8}$ (see e.g. [NSZ]).

Let $X_{\Delta}$ denote the variety of Borel subgroups of $G$. Then it is obvious that $R$ splits over $k\left(X_{\Delta}\right)$. On the other hand, the kernel of the Rost invariant is trivial modulo 3 by Prop. 10.21. Therefore the upper motives of $D$ and $X_{\Delta}$ are isomorphic. Thus, $J_{3}(G)=(1,0)$. By Lemma $10.22 X_{8}$ is isotropic over a field extension $L$ of degree coprime to 3 . But then $X_{7}$ is also isotropic over an extension of $L$ of degree dividing 2 .

Finally, (2) obviously implies (3), and (3) implies (2) by the classification of possible Tits indexes in [Ti66]. Property (2) easily implies (1). 
10.24. Remark. If one attempts to sharpen the theorem by deleting the text "over a field extension of degree coprime to 3 ", the implication $(2) \Rightarrow(1)$ still holds but $(1) \Rightarrow(2)$ fails. Indeed, for $\xi \in H^{1}\left(\mathbb{R}, G_{0}\right)$ such that ${ }_{\xi}\left(G_{0}\right)$ is the compact $\mathrm{E}_{8}$, $r_{G_{0}}(\xi)$ is zero.

Table 10C summarizes what we have proved about the relationship between the Rost invariant, $J$-invariant, and Tits indexes for groups of type $\mathrm{E}_{8}$ at the prime 3. Note that we proved the equivalent description for Tits indexes and the Rost invariant over fields of arbitrary characteristic, and the equivalent description for the $J$-invariant only for fields of characteristic 0 .

Note also that for every $k$, there is a versal torsor $\xi \in H^{1}\left(K, G_{0}\right)$ for some extension $K / k$, and that for such a $\xi, J_{3}(G)$ is maximal [PSZ, p. 1036], i.e., $(1,1)$. By Theorem 10.23, the 3 -torsion part of $r_{G_{0}}(\xi)$ is not a symbol in $H^{3}(L, \mathbb{Z} / 3 \mathbb{Z}(2))$ for every extension $L / K$ of degree not divisible by 3 .

\begin{tabular}{c|ccc}
$J_{3}(G)$ & $(0,0)$ & $(1,0)$ & $(1,1)$ \\
\hline $\begin{array}{c}\text { Tits index of } G \\
r_{G_{0}}(\xi)\end{array}$ & split & $1 . \smile \odot \multimap$ & anisotropic \\
0 & nonzero symbol & otherwise
\end{tabular}

TABLE 10C. Dictionary relating the mod-3 $J$-invariant of $G$, the Tits index of $G$ over a 3 -closure $K$ of $k$, and the Rost invariant of $r_{G_{0}}\left(\xi_{K}\right)$, for $G_{0}$ split of type $\mathrm{E}_{8}$.

10.25. Corollary. Let $C$ be a smooth projective irreducible curve over $\mathbb{Q}_{p}$ with $p \neq 3$. If $G$ is a group of type $\mathrm{E}_{8}$ over $\mathbb{Q}_{p}(C)$, then the $G$-variety $X_{7,8}$ is isotropic over a field extension of degree coprime to 3.

Proof. By [PaSu10, Theorem 3.5] each element in $H^{3}\left(\mathbb{Q}_{p}(C), \mathbb{Z} / 3\right)$ is a symbol over a field extension of degree coprime to 3 . Therefore by Theorem 10.23 the variety $X_{7,8}$ is isotropic over a field extension of degree coprime to 3 .

Acknowledgements. The authors sincerely thank Charles De Clercq, Wilberd van der Kallen, Geordie Williamson, Maksim Zhykhovich, and the referees for their remarks. The first author's research was partially supported by NSF grant DMS-1201542, NSA grant H98230-11-1-0178, Emory University, and the Charles T. Winship Fund. The second and the third authors gratefully acknowledge the support of the MPIM Bonn and of the SFB/Transregio 45 Bonn-Essen-Mainz. The second author was also supported by JSC "Gazprom Neft" and RFBR 13-01-91150, 13-01-00429, 13-01-92699.

\section{REFERENCES}

[BP] E. Bayer-Fluckiger and R. Parimala, Classical groups and the Hasse principle, Ann. Math. (2) 147 (1998), 651-693.

[Bri] M. Brion, Equivariant Chow groups for torus actions, Transf. Groups 2 (1997), no. 3, 225-267.

[Bro] P. Brosnan, Steenrod operations in Chow theory, Trans. Amer. Math. Soc. 355 (2003), no. 5, 1869-1903.

[C95] V. Chernousov, A remark on the mod 5-invariant of Serre for groups of type $\mathrm{E}_{8}$, Math. Notes 56 (1995), 730-733. Russian original: Mat. Zametki 56 (1994), no. 1, 116-121. 
[C10] - On the kernel of the Rost invariant for $\mathrm{E}_{8}$ modulo 3, In J.-L. Colliot-Thélène, S. Garibaldi, R. Sujatha, and V. Suresh, editors, Quadratic forms, linear algebraic groups, and cohomology, volume 18 of Developments in Mathematics, pages 199-214. Springer, 2010.

[CGM] V. Chernousov, S. Gille, A. Merkurjev, Motivic decomposition of isotropic projective homogeneous varieties, Duke Math. J. 126 (2005), no. 1, 137-159.

$[\mathrm{CM}] \quad$ V. Chernousov, A. Merkurjev, Motivic decomposition of projective homogeneous varieties and the Krull-Schmidt theorem, Transform. Groups 11 (2006), no. 3, 371-386.

[DC] Ch. De Clercq, A going down theorem for Grothendieck Chow motives, Quart. J. Math. 64 (2013), no. 3, 721-728.

[De] M. Demazure, Désingularisation des variétés de Schubert généralisées, Ann. Sci. École Norm. Sup. (4) 7 (1974), 53-88.

[DG] M. Demazure and A. Grothendieck, Schémas en groupes III: Structure des schemas en groupes reductifs, Lecture Notes in Mathematics, vol. 153, Springer, 1970.

[Du] H. Duan, Multiplicative rule of Schubert classes, Invent. Math. 159 (2005), no. 2, 407436; erratum, Invent. Math. 177 (2005), no. 3, 683-684.

[DuZ] H. Duan, X. Zhao, A unified formula for Steenrod operations in flag manifolds, Compos. Math. 143 (2007), no. 1, 257-270.

[EKM] R. Elman, N. Karpenko, and A. Merkurjev, The algebraic and geometric theory of quadratic forms, AMS Colloquium Publications, vol. 56, 2008

[FlOV] H. Flenner, L. O'Carroll, W. Vogel, Joins and intersections, Springer Monographs in Math., 1999.

[FoK] S. Fomin, A. Kirillov, The Yang-Baxter equation, symmetric functions, and Schubert polynomials, Discrete Math. 153 (1996), 123-143.

[Ga09a] S. Garibaldi, Orthogonal involutions on algebras of degree 16 and the Killing form of $\mathrm{E}_{8}$, with an appendix by K. Zainoulline, Quadratic forms - algebra, arithmetic, and geometry, Contemp. Math. 493 (2009), 131-162.

[Ga09b] Cohomological invariants: exceptional groups and spin groups, with an appendix by Detlev W. Hoffmann, Memoirs Amer. Math. Soc. 200 (2009), no. 937.

[GaPe] S. Garibaldi and H.P. Petersson, Wild Pfister forms over Henselian fields, K-theory, and conic division algebras, J. Algebra 327 (2011), 386-465.

[GaGi] S. Garibaldi and P. Gille, Algebraic groups with few subgroups, J. London Math. Soc. (2) 80 (2009), 405-430.

[GaQu] S. Garibaldi and A. Quéguiner-Mathieu, Restricting the Rost invariant to the center, St. Petersburg Math. J. 19 (2008), no. 2, 197-213.

[GaSe] S. Garibaldi and N. Semenov, Degree 5 invariant of E 8 , IMRN (2010), no. 19, 37463762 .

[GaMS] S. Garibaldi, A. Merkurjev, J.-P. Serre, Cohomological invariants in Galois cohomology, University Lecture Series 28, AMS, Providence, RI, 2003.

[GKM] M. Goresky, R. Kottwitz, R. MacPherson, Equivariant cohomology, Koszul duality, and the localization theorem, Invent. Math. 131 (1998), 25-83.

[Gi00] P. Gille, Invariants cohomologiques de Rost en caractéristique positive, K-Theory 21 (2000), 57-100.

[Gi01] Cohomologie galoisienne des groupes quasi-déployés sur des corps de dimension cohomologique $\leq 2$, Compos. Math. 125 (2001), no. 3, 283-325.

[GiSe] P. Gille and N. Semenov, Zero cycles on projective varieties and the norm principle, Compos. Math. 146 (2010), 457-464.

[GHZ] V. Guillemin, T. Holm, C. Zara, A GKM description of the equivariant cohomology ring of a homogeneous space, J. Algebraic Comb. 23 (2006), 21-41.

[HHH] M. Harada, A. Henriques, T. Holm, Computation of generalized equivariant cohomologies of Kac-Moody flag varieties, Advances in Mathematics 197 (2005), no. 1, 198-221.

[HHK] D. Harbater, J. Hartmann, D. Krashen, Local-global principles for Galois cohomology, Comment. Math. Helv. 89 (2014), no. 1, 215-253.

[JaW] B. Jacob, A.R. Wadsworth, Division algebras over Henselian fields, J. Algebra 128 (1990), 126-179.

[Ju] C. Junkins, The J-invariant and Tits algebras for groups of inner type $\mathrm{E}_{6}$, Manuscripta Math. 140 (2013), 249-261. 
[Ka95] N. Karpenko, Grothendieck Chow motives of Severi-Brauer varieties St. Petersburg Math. J. 7 (1996), 649-661 [Russian original: Algebra i Analiz 7 (1995), 196-213].

[Ka98] Codimension 2 cycles on Severi-Brauer varieties, K-Theory 13 (1998), no. 4, 305-330.

[Ka03] - On the first Witt index of quadratic forms, Invent. Math. 153 (2003), no. 2, 455-462.

[Ka04] Holes in $I^{n}$, Ann. Sci. Éc. Norm. Sup. 37 (2004), no. 6, 973-1002.

[Ka10] Hyperbolicity of orthogonal involutions, Doc. Math. extra volume: Andrei A. Suslin's Sixtieth Birthday (2010), 371-392.

[Ka13] Upper motives of algebraic groups and incompressibility of Severi-Brauer varieties, J. reine angew. Math. 677 (2013), 179-198.

$[\mathrm{KaM}] \quad$ N. Karpenko, A. Merkurjev, Canonical p-dimension of algebraic groups, Adv. Math. 205 (2006), no. 2, 410-433.

[KaZ] N. Karpenko, M. Zhykhovich, Isotropy of unitary involutions, Acta Math. 211 (2013), no. $2,227-253$.

[Kato 82] K. Kato. Galois cohomology of complete discrete valuation fields, volume 967 of Lecture Notes in Math., pages 215-238. Springer, 1982.

[Kato 86] — A Hasse principle for two-dimensional global fields, with an appendix by J.L. Colliot-Thélène, J. reine angew. Math. 366 (1986), 142-183.

[KMRT] M.-A. Knus, A. Merkurjev, M. Rost, and J.-P. Tignol, The Book of Involutions, AMS Colloquium Pub. 44, Providence, RI, 1998.

[KnT] A. Knutson, T. Tao, Puzzles and (equivariant) cohomology of Grassmannians, Duke Math. J. 119 (2003), no. 2, 221-260.

[Kö] B. Köck, Chow motif and higher Chow theory of $G / P$, Manuscr. Math. 70 (1991), 363-372.

[KonoI] A. Kono, K. Ishitoya, Squaring operations in mod 2 cohomology of quotients of compact Lie groups by maximal tori. Algebraic topology, Barcelona, 1986, 192-206, Lecture Notes in Math. 1298, Springer, Berlin, 1987.

[Lam] T.-Y. Lam, Introduction to quadratic forms over fields, Graduate Studies in Math. 67, American Mathematical Society, 2004.

[Leep] D. Leep, The u-invariant of p-adic function fields, J. reine angew. Math. 679 (2013), 65-73, doi:10.1515/crelle.2012.029.

[Ma] Y. Manin, Correspondences, motives and monoidal transformations, Matematicheskij Sbornik 77 (119) (1968), no. 4, 475-507 (in Russian). Engl. transl.: Math. USSR Sb. 6 (1968), 439-470.

[MPW] A. Merkurjev, I. Panin, A. Wadsworth, Index reduction formulas for twisted flag varieties I, K-theory 10 (1996), no. 6, 517-596.

[MT95] A. Merkurjev, J-P. Tignol, The multipliers of similitudes and the Brauer group of homogeneous varieties, J. reine angew. Math. 461 (1995), 13-47.

$[\mathrm{NeZ}] \quad$ A. Nenashev, K. Zainoulline, Oriented cohomology and motivic decompositions of relative cellular spaces, J. Pure Appl. Algebra 205 (2006), no. 2, 323-340.

[NSZ] S. Nikolenko, N. Semenov, K. Zainoulline, Motivic decomposition of anisotropic varieties of type $\mathrm{F}_{4}$ into generalized Rost motives, J. of K-theory 3 (2009), no. 1, 85-102.

$[\mathrm{PaPr}] \quad$ R. Parimala, R. Preeti, Hasse principle for classical groups over function fields of curves over number fields, J. Number Theory 101 (2003), no. 1, 151-184.

[PaSu98] R. Parimala, V. Suresh, Isotropy of quadratic forms over function fields of p-adic curves, Inst. Hautes Études Sci. Publ. Math. 88 (1998), 129-150.

[PaSu10] _ The u-invariant of the function fields of $p$-adic curves, Ann. Math. 172 (2010), 1391-1405.

[Pey] E. Peyre, Galois cohomology in degree three and homogeneous varieties, $K$-Theory 15 (1998), no. 2, 99-145.

[PS10] V. Petrov, N. Semenov, Generically split projective homogeneous varieties, Duke Math. J. 152 (2010), 155-173.

[PS12] _ , Generically split projective homogeneous varieties. II, J. of K-theory 10 (2012), issue 1, 1-8.

[PSZ] V. Petrov, N. Semenov, K. Zainoulline, J-invariant of linear algebraic groups, Ann. Sci. Éc. Norm. Sup. 41 (2008), 1023-1053. 
[PSt] V. Petrov, A. Stavrova, Tits indices over semilocal rings, Transf. Groups 16 (2011), 193-217.

[QSZ] A. Quéguiner-Mathieu, N. Semenov, K. Zainoulline, The J-invariant, Tits algebras and triality, J. of Pure and Applied Algebra 216 (2012), 2614-2628.

[Ro91] M. Rost, A (mod 3) invariant for exceptional Jordan algebras, C. R. Acad. Sci. Paris Sér. I Math. 313 (1991), 823-827.

[Ro99] A A descent property for Pfister forms, J. Ramanujan Math. Soc. 14 (1999), no. $1,55-63$.

[Ro07] On the basic correspondence of a splitting variety, Preprint 2007. Available from http://www.math.uni-bielefeld.de/ rost

[ScvB] A. Schofield, M. van den Bergh, The index of a Brauer class on a Brauer-Severi variety, Trans. Amer. Math. Soc. 333 (1992), 729-739.

[Sem] N. Semenov, Motivic construction of cohomological invariants, Preprint 2009. Available from http://arxiv.org/abs/0905.4384

[Sp98] T.A. Springer, Linear algebraic groups, 2nd ed., Progress in Math. 9, Birkhäuser Boston, 1998.

[Sp06] Some groups of type E $\mathrm{E}_{7}$, Nagoya Math. J. 182 (2006), 259-284.

[Ti66] J. Tits, Classification of algebraic semisimple groups, In Algebraic Groups and Discontinuous Subgroups (Proc. Symp. Pure Math.), Amer. Math. Soc., Providence, R.I., 1966, 33-62.

[Ti71] _ Représentations linéares irréductibles d'un groupe réductif sur un corps quelconque, J. reine angew. Math. 247 (1971), 196-220.

[Ti90] _ Strongly inner anisotropic forms of simple algebraic groups, J. Algebra 131 (1990), no. 2, 648-677.

[Ti92] Sur les degrés des extensions de corps déployant les groupes algébriques simples, C. R. Acad. Sci. Paris Sér. I Math., 315 (1992), no. 11, 1131-1138.

[Ty] J. Tymoczko, Divided difference operators for partial flag varieties, Preprint 2009, http: //arxiv.org/abs/0912.2545

[Vi03] A. Vishik, Motives of quadrics with applications to the theory of quadratic forms, Lect. Notes Math., 1835 (2003) 25-101.

[Vi07] Fields of u-invariant $2^{r}+1$, In: Algebra, Arithmetic and Geometry, Manin Festschrift, Birkhäuser (2007).

[Vi10] Excellent connections in the motives of quadrics, Ann. Sci. Éc. Norm. Sup. 44 (2010) 183-195.

[ViZ] A. Vishik, K. Zainoulline, Motivic splitting lemma, Doc. Math. 13 (2008), 81-96.

Garibaldi: Institute for Pure and Applied Mathematics, UClA, 460 Portola Plaza, Box 957121, Los Angeles, California 90095-7121, USA

E-mail address: skip@garibaldibros.com

Petrov: St. Petersburg Department of Steklov Mathematical Institute, Russian Academy of Sciences, Fontanka 27, 191023 St. Petersburg, Russia AND

Chebyshev Laboratory, St. Petersburg State University, 14th Line, 29b, 199178 St. PeTERSBURG, RusSiA

E-mail address: victorapetrov@googlemail.com

Semenov: Institut für Mathematik, Johannes Gutenberg-Universität Mainz, StaudingerWEG 9, D-55128 MAINZ, GERMANy

E-mail address: semenov@uni-mainz.de 